Aus der Abteilung Neurodegeneration und Neurorestaurationsforschung (Prof. Dr. med. J. B. Schulz)

im Zentrum Neurologische Medizin

der Medizinischen Fakultät der Universität Göttingen

\title{
Magnetresonanztomographie-basierte Volumetrie bei hereditären spinozerebellären Ataxien
}

\author{
INAUGURAL - DISSERTATION
}

\author{
zur Erlangung des Doktorgrades \\ der Medizinischen Fakultät \\ der Georg-August-Universität zu Göttingen \\ vorgelegt von \\ Johannes Borkert \\ aus Hamburg
}


D e k a n:

Prof. Dr. med. C. Frömmel

I. Berichterstatter:

Prof. Dr. med. J. B. Schulz

II. Berichterstatter/in:

III. Berichterstatter/in:

Tag der mündlichen Prüfung: 


\section{Inhaltsverzeichnis}

1.1 Allgemeines

1.2 Spinozerebelläre Ataxie 1,3 und $6 \quad 3$

1.2.1 Epidemiologie 3

1.2.2 Pathogenese 3

1.2.3 Neuropathologie 4

1.2.4 Klinik 5

$\begin{array}{lll}1.2 .5 & \text { Therapie } & 7\end{array}$

$\begin{array}{lll}1.3 & \text { Magnetresonanztomographie } & 7\end{array}$

1.4 Zielsetzung 9

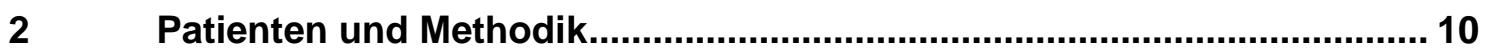

$\begin{array}{lll}2.1 & \text { Kontrollgruppe } & 10\end{array}$

2.2 Patientenkollektiv 10

2.3 Methodik 10

2.3.1 MR-Bildgebung 10

2.3.2 Volumetrie 11

$2.4 \quad$ Klinische Parameter 17

$\begin{array}{lll}2.5 & \text { Repeatlängen } & 18\end{array}$

2.6 Datenanalyse 18

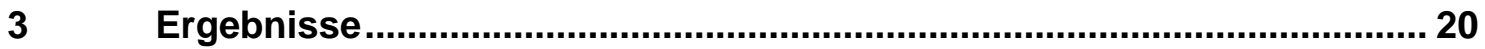

3.1 Charakteristika der Kontrollgruppe und des Patientenkollektivs 20

$\begin{array}{lll}3.2 & \text { Volumetrie } & 21\end{array}$

$\begin{array}{lll}3.3 & \text { Korrelationen } & 27\end{array}$

3.3.1 Volumina, korreliert mit den klinischen Symptomen (SARA) 27

3.3.2 Volumina, korreliert mit den CAG-Repeatlängen 28

3.3.3 Volumina, korreliert mit der Erkrankungsdauer 29

3.3.4 Regressionsanalyse für die Varianz von SARA 30

3.4 Klassifizierung der Gruppen mit Hilfe der Diskriminanzanalyse 30

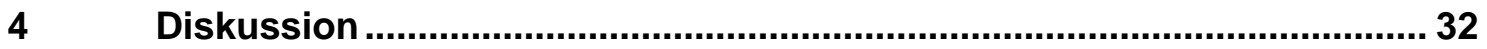

4.1 MR-Bildgebung mit anschließender 3D-Volumetrie 32

4.2 Demographische und klinische Charakteristika 34

$4.3 \quad$ Neuroanatomische Veränderungen bei SCA1, SCA3 und SCA6 34

4.4 Klinisch-neuroanatomische Korrelationen und Krankheitsverlauf 35

$\begin{array}{lll}4.5 & \text { Prädiktive Statistik } & 37\end{array}$

$\begin{array}{lll}4.6 & \text { Schlussfolgerung und Ausblick } & 38\end{array}$

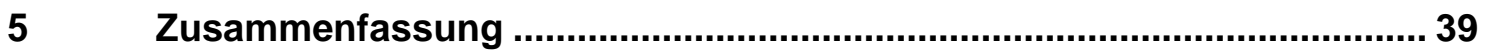

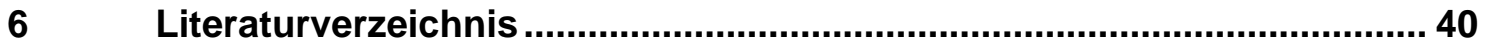




\section{Abkürzungsverzeichnis}

$\begin{array}{ll}\text { ADCA } & \text { Autosomal-dominant zerebelläre Ataxie } \\ \text { ANOVA } & \text { Analysis of variance } \\ \text { CAG } & \text { Cytosin, Adenin, Guanin } \\ \text { CCA } & \text { Zerebellärkortikale Atrophie } \\ \text { CT } & \text { Computertomographie } \\ \text { DRPLA } & \text { Dentato-rubro-pallido-luysische Atrophie } \\ \text { EA } & \text { Episodische Ataxie } \\ \text { EuroSCA } & \text { European integrated project on spinocerebellar ataxias } \\ \text { ICARS } & \text { International cooperative ataxia rating scale } \\ \text { IQR } & \text { Interquartile range } \\ \text { LSD } & \text { Least significant difference } \\ \text { MJD } & \text { Machado-Joseph disease } \\ \text { MRT } & \text { Magnetresonanztomographie } \\ \text { OPCA } & \text { Olivo-pontino-zerebelläre Atrophie } \\ \text { RG } & \text { Region-growing } \\ \text { ROI } & \text { Region of interest } \\ \text { SARA } & \text { Scale for the assessment and rating of ataxia } \\ \text { SCA } & \text { Spinozerebelläre Ataxie } \\ \text { TICV } & \text { Totales intrakranielles Volumen }\end{array}$




\section{$1 \quad$ Einleitung}

\section{$1.1 \quad$ Allgemeines}

Spinozerebelläre Ataxien (SCA) gehören der genetisch und klinisch heterogenen Gruppe der autosomal-dominanten zerebellären Ataxien (ADCA) an. Charakteristisch sind eine progressive Ataxie, Dysarthrie und Nystagmus, die alle durch eine Degeneration des Zerebellums und seiner afferenten und efferenten Bahnen bedingt sind. Darüber hinaus bestehen oft auch Veränderungen im Hirnstamm, in den Basalganglien, im Rückenmark, in der Retina und im peripheren Nervensystem.

Anhand neuropathologischer Kriterien wurden die zerebellären Ataxien zunächst von Holmes 1908 und später von Greenfield 1954 beschrieben und klassifiziert. Beide unterschieden zwischen zerebellären, spinozerebellären und olivo-pontino-zerebellären Degenerationsformen. Eine Einteilung, die den unterschiedlichen Erscheinungsbildern der dominant vererbten Ataxien Rechnung trug und auch heute noch Verwendung findet, wurde von Harding 1982 vorgestellt. Charakteristisch für den häufigsten Typ, ADCA-I, ist eine progressive Ataxie in Kombination mit zusätzlichen Symptomen, wie Sakkadenverlangsamung / Ophthalmoplegie, Pyramidenbahnzeichen, Muskelatrophien, Basalgangliensymptomen und Sensibilitätsstörungen. Bei der ADCA-II liegt eine progressive Ataxie in Kombination mit pigmentärer Retinadegeneration vor. Kennzeichen für die ADCA-III ist eine progressive rein zerebelläre Ataxie ohne zusätzliche Symptome. Die progressiv verlaufenden ADCAs werden heute überwiegend genetisch als SCA bezeichnet. Sie werden abgegrenzt von den sehr viel selteneren, ebenfalls autosomal-dominant vererbten episodischen Ataxien (EA).

Pathogenetisch liegt vielen der bis heute identifizierten SCA-Mutationen eine CAGRepeat-Expansion zugrunde. Die SCAs gehören somit, genauso wie die HuntingtonKrankheit, die spinobulbäre Muskelatrophie und die dentato-rubro-pallido-luysische Atrophie (DRPLA), zur Gruppe der Polyglutamin-Krankheiten. Bis auf einige Ausnahmen gelten für die Polyglutamin-Krankheiten folgende Charakteristika (Stevanin et al. 2000; Klockgether 2005): (1) Krankheitsbeginn meist im Erwachsenenalter, (2) progressiver Krankheitsverlauf, (3) klinische Symptome treten erst ab einer bestimmten Anzahl von CAG-repeats auf, (4) es existiert eine negative Korrelation zwischen der 
Anzahl der CAG-repeats und dem Krankheitsbeginn, (5) die Anzahl der CAG-repeats ist instabil und nimmt in der Tendenz von Generation zu Generation zu (dieses Phänomen wird als Antizipation bezeichnet und tritt bei der SCA6 nicht auf) und (6) der kodierende Genabschnitt wird ubiquitär exprimiert.

Das diagnostische Vorgehen bei Patienten mit dem Syndrom Ataxie besteht zunächst aus einer sorgfältigen Anamneseerhebung. Insbesondere die Familienanamnese kann bei Patienten mit dem Leitsymptom Ataxie wertvolle Hinweise auf einen eventuell zugrunde liegenden dominanten oder rezessiven Erbgang liefern (Klockgether 2005; Klockgether 2008). Im Anschluss an die klinische Untersuchung ist eine Magnetresonanztomographie (MRT) des Gehirns aus folgenden Gründen empfehlenswert: (1) Ausschluss anderer Differenzialdiagnosen wie bspw. zerebrale Gefäßanomalien, Malignome oder Multiple Sklerose und (2) Quantifizierung von Muster und Grad der Atrophie. Dieses diagnostische Vorgehen ermöglicht zwar keine konkrete Klassifizierung, kann aber das Spektrum der in Frage kommenden kausalen Mutationen einschränken. Die abschließende molekulargenetische Untersuchung, die unter Berücksichtigung der zuvor gewonnenen Erkenntnisse gezielter erfolgen kann, ermöglicht in einer Vielzahl der Fälle eine zweifelsfreie Feststellung der zugrunde liegenden Mutation. Bei ca. $30 \%$ ist bis heute jedoch eine exakte genetische Diagnostik nicht möglich.

Nach diagnostizierter SCA ist es mit Hilfe der MRT außerdem möglich, atrophische Veränderungen und das Fortschreiten der Erkrankung im Verlauf zu dokumentieren (Döhlinger et al. 2008). Allerdings konnte der Zusammenhang zwischen dem Ausmaß der Atrophie einzelner neuroanatomischer Strukturen einerseits und der Erkrankungsdauer, dem klinischen Erscheinungsbild und der Anzahl der CAG-repeats andererseits noch nicht bestätigt werden. Vielmehr existieren einige Studien mit widersprüchlichen Resultaten (Onodera et al. 1998; Klockgether et al. 1998b; Guerrini et al. 2004; Ying et al. 2006). Aufgrund des langsamen Fortschreitens der SCA wäre es aber wünschenswert, wenn die MRT als zuverlässiger Marker für die Wirksamkeit der medikamentösen Therapie dienen könnte. Um den Stellenwert der MRT bei Patienten mit SCA genauer zu bestimmen, wurde daher innerhalb des europäischen Konsortiums EuroSCA eine Multizenterstudie durchgeführt. 


\subsection{Spinozerebelläre Ataxie 1, 3 und 6}

\subsubsection{Epidemiologie}

Die Prävalenz der ADCA wird weltweit auf 1:100.000 geschätzt (Schoenberg 1978). In Mitteleuropa beträgt die Prävalenz für die SCA etwa 3:100.000. 70\% davon entfallen auf die häufigsten Mutationen SCA1, SCA2, SCA3 und SCA6 (Klockgether et al. 1998a; Schöls et al. 2004). SCA3, auch bekannt als Machado-Joseph disease (MJD), ist der weltweit und auch in Deutschland am meisten verbreitete Genotyp (Murata et al. 1998a).

\subsubsection{Pathogenese}

Die betroffenen Gene und die ursächlichen Mutationen der SCA1, SCA3 und SCA6 wurden bereits identifiziert (Orr et al. 1993; Takiyama et al. 1993; St George-Hyslop et al. 1994; Vahedi et al. 1995). Die Produkte der SCA1- und SCA3-Gene sind Proteine unbekannter Funktion. Sie kommen ubiquitär vor und werden als Ataxin 1 bzw. Ataxin 3 bezeichnet. Die Funktion des SCA6-Gens hingegen ist bekannt. Es kodiert eine spannungsabhängige Untereinheit des Kalziumkanals CACNA1A. Dieser wird in erster Linie in Purkinje-Zellen des Zerebellums exprimiert (Zhuchenko et al. 1997).

Tabelle 1 CAG-Repeatlängen der SCA1, SCA3 und SCA6 (Bürk et al. 1999, S. 492)

\begin{tabular}{|l|c|c|}
\hline & physiologisch & pathologisch \\
\hline SCA1 & $6-39$ & $40-83$ \\
\hline SCA3 & $14-40$ & $62-84$ \\
\hline SCA6 & $4-16$ & $21-27$ \\
\hline
\end{tabular}

Bei allen Mutationen handelt es sich um ungewöhnlich lange, instabile Wiederholungen von drei Nukleotiden, den Basen Cytosin, Adenin und Guanin (kurz: CAG-repeat). Die Basensequenz CAG kodiert für die Aminosäure Glutamin. Im translatierten Protein wird demzufolge ein pathologisch verlängerter Polyglutaminstrang eingebaut. Die Tabelle 1 gibt Aufschluss über die physiologischen und die expandierten Repeatzahlen in Abhängigkeit von der zugrunde liegenden Erkrankung (Bürk et al. 1999). 
Zwischen der Anzahl der CAG-Wiederholungen und dem Krankheitsbeginn besteht eine inverse Korrelation. Patienten mit einer verhältnismäßig niedrigen Anzahl an CAG-Wiederholungen erkranken später als Patienten mit einer hohen Anzahl (Bürk et al. 1996; van de Warrenburg et al. 2005). Für die SCA3 wird darüber hinaus von einer positiven Korrelation zwischen CAG-Wiederholungen und dem klinischen Fortschreiten der Krankheit berichtet (Klockgether et al. 1998a).

\subsubsection{Neuropathologie}

Neuropathologisch findet sich bei den SCA immer Gliose und ein Untergang von Purkinje-Zellen (Klockgether 1999, 343-361; Bürk et al. 1999). Das Muster der neurodegenerativen Veränderungen von Hirnstamm, Basalganglien, Retina und peripherem Nervensystem variiert in Abhängigkeit von der zugrunde liegenden Mutation. Spezifisch für einen einzelnen Genotyp ist es jedoch nicht. Es lässt sich auch nicht mit der selektiven Expression der Genprodukte erklären.

Die MRT bei SCA1 zeigt Atrophie des zerebellären Kortex, des Pons und der Oliven (Abb. 1a). Dies ist vereinbar mit der Diagnose einer olivo-pontino-zerebellären Atrophie (OPCA) (Bürk et al. 1996; Klockgether et al. 1998b; Guerrini et al. 2004).
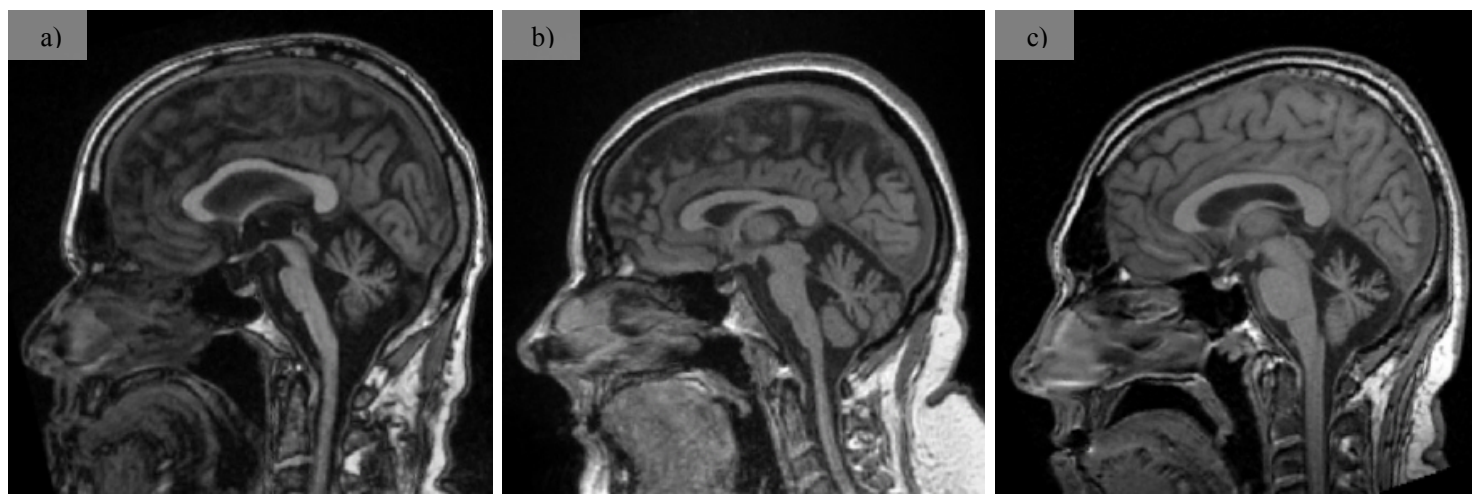

Abb. 1 T $\mathrm{T}_{1}$-gewichtete MRT-Bilder (Mediansagittalschnitt): a) MRT eines 63-jährigen SCA1Patienten, b) MRT einer 40-jährigen SCA3-Patientin, c) MRT eines 43-jährigen SCA6-Patienten.

Neurodegenerative Veränderungen sind bei SCA3 im Vermis, in den zerebellären Hemisphären und im Hirnstamm festzustellen (Abb. 1b). Das Atrophiemuster ähnelt damit dem von SCA1, wenngleich es weniger stark ausgeprägt ist (Bürk et al. 1996; Klockgether et al. 1998b). Darüber hinaus fanden Klockgether und seine Kollegen eine 
Atrophie im Nucleus caudatus (Klockgether et al. 1998b). Murata und seine Kollegen beobachteten ferner eine Volumenabnahme in den oberen Kleinhirnstielen, im Frontalund Temporallappen sowie eine Verminderung des Längsdurchmessers des Globus pallidus (Murata et al. 1998a).

Für SCA6 zeigt die zerebrale Bildgebung eine schwere Atrophie des Vermis und eine milde Atrophie der zerebellären Hemisphären (Abb. 1c). Atrophien für den Pons, die mittleren Kleinhirnstiele oder andere Strukturen der hinteren Schädelgrube konnten nicht festgestellt werden (Satoh et al. 1998; Schöls et al. 1998a; Butteriss et al. 2005). Bei zwei Patienten fanden Satoh und seine Kollegen eine signifikante Volumenabnahme des zerebralen Kortex (Satoh et al. 1998). Murata und seine Kollegen beschrieben eine milde Atrophie des anteroposterioren Ponsdurchmessers und der Durchmesser der mittleren Kleinhirnstiele (Murata et al. 1998b).

\subsubsection{Klinik}

Der Krankheitsbeginn für SCA1 und SCA3 liegt in der Regel zwischen dem 35. und 45. Lebensjahr (Klockgether et al. 1998a). Bei Patienten mit SCA6 sind die ersten Symptome häufig erst zu Beginn der fünften Lebensdekade zu erwarten (Stevanin et al. 1997; Schöls et al. 1997). Rollstuhlpflichtig werden die Patienten im Mittel 15 Jahre nach Feststellung der ersten Symptome. Der Tod tritt ca. 25 Jahre nach Krankheitsbeginn ein. In Bezug auf den Krankheitsbeginn wird in SCA1- und SCA3-Familien das Phänomen der Antizipation, d.h. spätere Generationen erkranken jünger, beobachtet (Bürk et al. 1999).

Gemeinsame Merkmale der SCA1, SCA3 und SCA6 sind progrediente Ataxie, Dysarthrie und Nystagmus (Klockgether et al. 1998a). SCA1 und SCA3, die zur Gruppe der ADCA-I gehören, werden von einer Reihe nicht zerebellärer Symptome, wie etwa supranukleärer Blickparese, Optikusatrophie, Basalgangliensymptome, Spastik, Demenz, sensible Störungen und Muskelatrophie, begleitet (Klockgether 2005). Die klinische Diagnose einzelner Subtypen ist schwierig. Zum einen überschneiden sich die Phänotypen unterschiedlicher Genotypen. Zum anderen wird eine große Variabilität in der Ausprägung einzelner Symptome beobachtet (Schöls et al. 2004). 
Die Symptome bei SCA1 sind sehr variabel. Charakteristisch ist die Ataxie des Gangs, der Körperhaltung und der Gliedmaßen. Dysarthrie und Dysfunktion des okulomotorischen Systems, wie Blickrichtungsnystagmus, Sakkadendysmetrie, abgeschwächter vestibulo-okulärer Reflex und ein verminderter optokinetischer Nystagmus, sind ebenfalls zu beobachten. Im fortgeschrittenen Krankheitsverlauf können zusätzlich nicht zerebelläre Symptome wie eine Ophthalmoparese, Pyramidenbahnzeichen (Spastik, Hyperreflexie, Babinski-Zeichen), langsame Sakkaden und Dysphagie hinzukommen. Amyotrophie, Hyperkinesien und Sensibilitätsverlust sind hingegen eher selten (Sasaki et al. 1996; Schöls et al. 2004).

SCA3 ist phänotypisch sehr variabel. Die meisten Patienten zeigen typische spinozerebelläre Symptome, ähnlich wie bei der SCA1. Fernerhin sind SCA3-Patienten mit isolierter zerebellärer Ataxie, Parkinsonsyndrom, spastischer Paraplegie, Neuropathie oder einem Restless-legs-Syndrom beschrieben worden (Schöls et al. 2004). Schlafstörungen sind bei SCA3 nicht selten und werden mit dem Restless-legs-Syndrom in Verbindung gebracht (Schöls et al. 1998b). Pseudoexophthalmus, faziolinguale Myokymie und Dystonie werden als charakteristisch für SCA3 beschrieben, spezifisch sind sie jedoch nicht (Lima und Coutinho 1980).

Charakteristikum für SCA6 ist neben dem relativ späten Krankheitsbeginn die isolierte zerebelläre Ataxie. In einigen Fällen wird außerdem von einer milden peripheren Neuropathie, Bradykinesien, Dystonien, lebhaften Reflexen und positiven Pyramidenbahnzeichen berichtet (Schöls et al. 1998a). 


\subsubsection{Therapie}

Eine kurative Therapie für die SCA gibt es derzeit nicht. Auch wenn es keinen wissenschaftlichen Nachweis der Wirksamkeit gibt, sprechen Intuition und klinische Erfahrung für die Anwendung von Physiotherapie, Logopädie und Ergotherapie (Klockgether 2005). Darüber hinaus gibt es weitere symptomatische Behandlungsansätze. Yabe und seine Kollegen berichten von einer Verbesserung der Ataxie bei SCA6-Patienten nach der Gabe von Azetazolamid (Yabe et al. 2001). Sollte ein Parkinsonsyndrom bei SCA3-Patienten zur Funktionsbeeinträchtigung führen, hilft häufig eine dopaminerge Therapie. Diese wird in der Regel auch SCA3-Patienten mit einem Restless-legsSyndrom empfohlen (Abele et al. 2001).

\subsection{Magnetresonanztomographie}

Das Prinzip der Magnetresonanz wurde durch Purcell und seine Kollegen im Jahr 1946 entdeckt (Purcell et al. 1946). Die ersten MRT-Bilder wurden allerdings erst 1973 erzeugt (Lauterbur 1973). Seit Anfang der 1980er Jahre wird die MRT zunehmend in der klinischen Diagnostik eingesetzt. Im Unterschied zur Computertomographie (CT) wird das Bild nicht durch Röntgenstrahlen, sondern mit Hilfe eines starken Magnetfeldes und von Hochfrequenzimpulsen (Radiowellen) erzeugt. Ob ein Bild eher $\mathrm{T}_{1}$ - oder $\mathrm{T}_{2}$-gewichtet ist, hängt von der Wahl der Messparameter TR (Repetitionszeit), TE (Echozeit) und TI (Inversionszeit) ab.

Im Gegensatz zur CT besteht bei der MRT die Möglichkeit, Schichtebenen beliebiger Orientierung direkt, d.h. ohne Sekundärrekonstruktion, zu messen. Darüber hinaus ermöglicht das MRT insbesondere im Gehirn eine bessere Beschreibung der Gewebezusammensetzung. Eine Vielzahl von vergleichenden Untersuchungen hat gezeigt, dass die MRT der CT bei Fragestellungen nach Prozessen im Gehirn überlegen ist (Bydder et al. 1982; Brant-Zawadzki et al. 1983; Bradley et al. 1984). Das hohe Auflösungsvermögen der MRT ist besonders hilfreich für die Beurteilung des Zerebellums, des Hirnstamms und des Rückenmarks (Courchesne et al. 1989; Press et al. 1989). Nabatame und seine Kollegen konnten das Konzept von Greenfield, die Dichotomie zwischen olivo-pontino-zerebellärer (OPCA) und zerebellärkortikaler Atrophie (CCA), mit Hilfe der MRT bestätigen (Nabatame et al. 1988). 
Mittlerweile hat sich die MRT als Verlaufsparameter und als Biomarker für die Differentialdiagnose bei der Alzheimer-Demenz (Fox et al. 2001; Fox und Schott 2004), der Huntington-Erkrankung (Bamford et al. 1995; Rosas et al. 2001; Kassubek et al. 2004) und den akinestisch rigiden Bewegungsstörungen (Schulz et al. 1999; Gröschel et al. 2004; Schocke et al. 2004; Specht et al. 2005; Nicoletti et al. 2006) etablieren können.

In einem Review stellten Döhlinger und ihre Kollegen (Döhlinger et al. 2008) fest, dass quantitative Untersuchungen bei SCA bis heute nur bei einer geringen Anzahl an Betroffenen durchgeführt wurden (Klockgether et al. 1998b; Onodera et al. 1998; Guerrini et al. 2004; Ying et al. 2006). Abgesehen von einer Studie mit SCA3-Patienten (Onodera et al. 1998) konnte der Zusammenhang zwischen der CAG-Repeatlänge und dem Ausmaß der Atrophie bisher nicht bestätigt werden. Darüber hinaus wurde die Frage, ob Atrophie bestimmter neuroanatomischer Strukturen mit der klinischen Dysfunktion korreliert, lediglich in zwei Studien mit widersprüchlichen Resultaten untersucht. Guerrini und ihre Kollegen berichten von 28 Patienten, bei denen die Atrophie des Hirnstamms mit dem klinischen Erscheinungsbild, quantifiziert durch die International cooperative ataxia rating scale (ICARS), für SCA1, nicht aber jedoch für SCA2, korreliert (Guerrini et al. 2004). Ying und ihre Kollegen hingegen beschreiben einen Zusammenhang der pontinen und zerebellären Atrophie mit der klinischen Dysfunktion, quantifiziert mit Hilfe einer neurologischen Skala für die Einschätzung der Friedreich-Ataxie (Subramony et al. 2005), für SCA2 (Ying et al. 2006).

Nach wie vor ist nicht geklärt, ob die MRT die Differenzierung einzelner Genotypen ermöglicht. Der Nutzen der MRT als Biomarker für die Voraussage der klinischen Dysfunktion und des Krankheitsverlaufs ist ebenfalls noch nicht ausreichend bewiesen. Gründe hierfür sind die geringe Anzahl an Patienten in vorangegangenen Studien, ein fehlender direkter Vergleich verschiedener Genotypen, der Mangel an exakten dreidimensionalen Volumetrietechniken und - bis unlängst - das Fehlen von objektiven Beurteilungsskalen. Vor kurzem aber konnte sowohl die ICARS (Schmitz-Hübsch et al. 2006a) als auch die neu entwickelte Scale for the assessment and rating of ataxia (SARA) (Schmitz-Hübsch et al. 2006b) für den klinischen Einsatz bei ADCA validiert werden. 


\section{$1.4 \quad$ Zielsetzung}

Die MRT ermöglicht bereits in vivo eine gute, dreidimensionale Beurteilung neuroanatomischer Strukturen. Bei Patienten mit SCA ist daher bereits zu Lebzeiten und zu unterschiedlichen Zeitpunkten während des Erkrankungsprozesses ein Vergleich der neurodegenerativen Veränderungen mit den klinischen Symptomen möglich, während post-mortem-Untersuchungen in der Regel nur das Endstadium der Erkrankung abbilden. Um den Stellenwert der MRT bei der Differenzierung verschiedener SCAGenotypen und den Nutzen als Biomarker genauer zu quantifizieren, wurden innerhalb des europäischen Konsortiums EuroSCA 82 Patienten mit SCA1, SCA3 und SCA6 sowie 31 Kontrollpersonen im Rahmen einer Multizenterstudie rekrutiert. In der vorliegenden Arbeit wurde die MRT mit anschließender Volumetrie eingesetzt, um die folgenden Fragen zu beantworten:

(1) Existieren in Hinblick auf die neurodegenerativen Veränderungen Unterschiede zwischen SCA1, SCA3 und SCA6?

(2) Korrespondiert das klinische Erscheinungsbild (SARA) mit dem Muster der Atrophie?

(3) Welche Zusammenhänge bestehen zwischen CAG-Repeatlängen, Erkrankungsdauer und dem Ausmaß der Atrophie?

(4) Ist eine Klassifizierung der SCA1, SCA3 und SCA6 anhand des individuellen neuropathologischen Atrophiemusters in vivo möglich?

(5) Welchen Stellenwert hat die MRT in Hinblick auf die Wertigkeit als Verlaufsparameter? 


\section{Patienten und Methodik}

\section{$2.1 \quad$ Kontrollgruppe}

Die Kontrollgruppe umfasste 31 gesunde Personen, die eine ähnliche Alters- und Geschlechterverteilung (14 männliche, 17 weibliche, Durchschnittsalter $=49,8 \pm 16,2$ Jahre, Spannweite 16 - 76 Jahre) wie das Patientenkollektiv besaßen. Keiner der Probanden besaß eine neurologische Vorgeschichte. Die neurologischen Eingangsuntersuchungen zeigten keine Auffälligkeiten. Die Begutachtung der $\mathrm{T}_{1}$ - und $\mathrm{T}_{2}$-gewichteten Bilder durch einen erfahrenen Neuroradiologen ergab keine pathologischen Befunde.

\subsection{Patientenkollektiv}

Im Rahmen einer Multizenterstudie wurden 82 Patienten (SCA1: $n=48$, SCA3: $n=24$, SCA6: $\mathrm{n}=10$ ) aus acht Zentren des europäischen Konsortiums EuroSCA (Bonn, Brüssel, Essen, Mailand, Nijmegen, Paris, Tübingen und Warschau) rekrutiert. Einschlusskriterien waren (1) progressive Ataxie und (2) molekulare Diagnose einer SCA1-, SCA3oder SCA6-Mutation. Die Zusammenfassung der demographischen und klinischen Charakteristika findet sich in Tabelle 3.

Die Einwilligungserklärungen der Patienten und der gesunden Kontrollpersonen zur Studienteilnahme lagen vor. Das Studiendesign und die Durchführung der Studie wurden von den lokalen Ethikkommissionen genehmigt.

\subsection{Methodik}

\subsubsection{MR-Bildgebung}

Die Datenaufnahme erfolgte jeweils in einem der acht teilnehmenden Zentren des europäischen Konsortiums EuroSCA im Zeitraum von August 2005 bis August 2006. Die dreidimensionalen, $\mathrm{T}_{1}$-gewichteten MRT-Bilder wurden jeweils auf einem konventionellen 1,5-Tesla-Kernspintomographen mit einer Standard-Kopfspule erstellt. Für jedes Scannermodell der verschiedenen Firmen wurde ein separates MRT-Protokoll erstellt. Dieses Protokoll wurde in Hinblick auf Vergleichbarkeit und Reproduzierbarkeit mit Hilfe eines Phantoms überprüft. 
Die genauen Parameter sind in Tabelle 2 aufgeführt. Während der Messung waren die Probanden angehalten, die Augen zu schließen. Die erstellten 3D- $\mathrm{T}_{1}$-Datensätze wurden anschließend anonymisiert, auf CD archiviert und nach Göttingen geschickt, wo sie volumetrisch ausgewertet wurden.

Tabelle 2 Parameter für MR-Bildgebung

\begin{tabular}{|l|l|l|l|l|}
\hline & Siemens Sonata & Siemens Vision & Philipps & GE \\
\hline Sequence & MPRAGE & MPRAGE & MPRAGE & SPGR \\
\hline TR & 15 & 11.6 & 15 & 2500 \\
\hline TE & 5 & 4.69 & 5 & minimum \\
\hline TI & - & - & - & 500 \\
\hline Matrix & $256 \times 256$ & $256 \times 256$ & $256 \times 256$ & $256 \times 256$ \\
\hline Field of View & $230 \mathrm{~mm}$ & $230 \mathrm{~mm}$ & $230 \mathrm{~mm}$ & $230 \mathrm{~mm}$ \\
\hline Slice thickness & $1 \mathrm{~mm}$ & $1 \mathrm{~mm}$ & $1 \mathrm{~mm}$ & $1 \mathrm{~mm}$ \\
\hline Voxel size & $1 \times 1 \times 1 \mathrm{~mm}$ & $1 \times 1 \times 1 \mathrm{~mm}$ & $1 \times 1 \times 1 \mathrm{~mm}$ & $1 \times 1 \times 1 \mathrm{~mm}$ \\
\hline
\end{tabular}

\subsubsection{Volumetrie}

Die volumetrische Auswertung der MRT-Datensätze wurde von dem Autor dieser Dissertation anonym und ohne Kenntnis der Diagnose vorgenommen. Alle 3DDatensätze wurden zunächst mittels SPM5 vom DICOM in das ANALYZE-Format konvertiert. Unter Zuhilfenahme der lizenzfreien Software MRIcro folgte das AC-PC (Anteriore Commissur - Posteriore Commissur)-Alignment. Die Volumetrie wurde mit Hilfe einer in MATALB programmierten Software (Schulz et al. 1999; Gröschel et al. 2004; Hauser et al. 2006) in semiautomatischer Segmentierungstechnik durchgeführt: manuelle Markierung anatomischer Landmarken gefolgt von einem automatischen Region-growing Algorithmus (3D-RG). Die Reliabilität von Volumetrie-Techniken im allgemeinen, und die semiautomatische Region-growing Technik (vgl. Abschnitt 2.3.2.3) im speziellen wurde bereits an anderer Stelle untersucht (Luft et al. 1996; Luft et al. 1998). 


\subsubsection{Segmentierung der Regions of interest}

Das manuelle Segmentieren der Regions of interest (ROIs) bestand aus einer groben Vorsegmentierung, die sich an anatomischen Landmarken orientierte (Abb. 2). Dies war insbesondere an den Stellen notwendig, an denen das Auswertungsprogramm aufgrund unzureichender Intensitätsunterschiede nicht in der Lage war, die Grenzen der anatomischen Strukturen automatisch zu erkennen bzw. da, wo die Grenzen nicht durch unterschiedliche Grauwerte, sondern durch anatomische Konventionen (z.B. Mesenzephalon - Pons - Medulla oblongata) definiert waren.

Das Volumen jeder einzelnen Struktur erstreckte sich in der Regel über mehrere MRT-Schichten, so dass für jede Schicht eine separate ROI bestimmt werden musste. Folgende anatomische Strukturen wurden manuell segmentiert: Hirnstamm (Mesenzephalon, Pons, Medulla oblongata), Zerebellum (Hemisphären, Vermis), Basalganglien (Putamen, Nucleus caudatus) und Großhirnlappen (Frontal-, Temporal-, Parietal- und Okzipitallappen).

\section{Hirnstamm}

Als Begrenzung des Hirnstamms dienten drei Ebenen, deren Position sich an anatomischen Landmarken orientierte (Abb. 2). In mediosagittaler Einstellung wurde zunächst eine Ebene durch die Corpora mammillaria und die Commissura posterior gelegt.
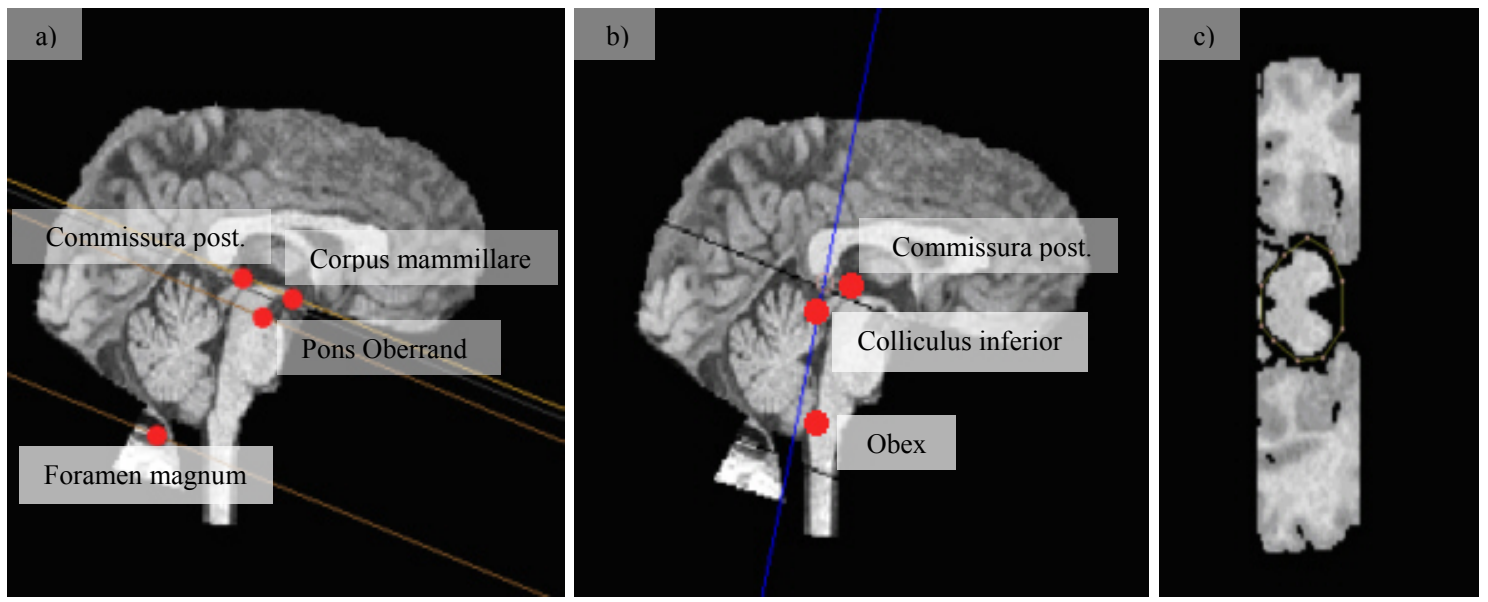

Abb. 2 Manuelle Segmentierung des Hirnstamms: a) anatomische Landmarken zur Erstellung von kranialer und kaudaler Begrenzung, b) Konstruktion der dorsalen Begrenzung des Hirnstamms, c) manuelles Nachsegmentieren des Hirnstamms in axialer Schnittführung. 
Diese Ebene wurde um 1/3 der Strecke zum kranial-anterioren Rand des Pons parallel nach kaudal verschoben und bildete somit die kraniale Begrenzung. Die kaudale Begrenzung entstand aus einer Parallelverschiebung der kranialen Begrenzung. Diese Ebene wurde soweit nach kaudal verschoben, bis ein Schnittpunkt mit dem dorsalen Rand des Foramen magnum resultierte. Eine Ebene, die die Commissura posterior und den Obex schnitt, wurde anschließend soweit parallel verschoben, bis sie dem Colliculus inferior von dorsal her anlag. Sie bildete nun die dorsale Begrenzung des Hirnstamms. Die Lage der dorsalen Ebenen hatte zur Folge, dass die mittleren Kleinhirnstiele später zum Volumen des Hirnstamms und nicht des Zerebellums hinzugerechnet wurden. Für die weitere Segmentierung des Hirnstamms in Mesenzephalon, Pons und Medulla oblongata wurden zwei Ebenen, parallel zur Corpora-mammillariaCommissura-posterior-Linie, erstellt. Als anatomische Landmarken dienten der kraniale und kaudale Ponsrand.

\section{Zerebellum}

Vorab wurde das Volumen des Hirnstamms vom Ausgangsdatensatz subtrahiert. Somit konnte sichergestellt werden, dass Voxel, die sich an den Übergangsbereichen zwischen Hirnstamm und Zerebellum befanden, bei der Volumenberechnung nur einmal berücksichtigt wurden.
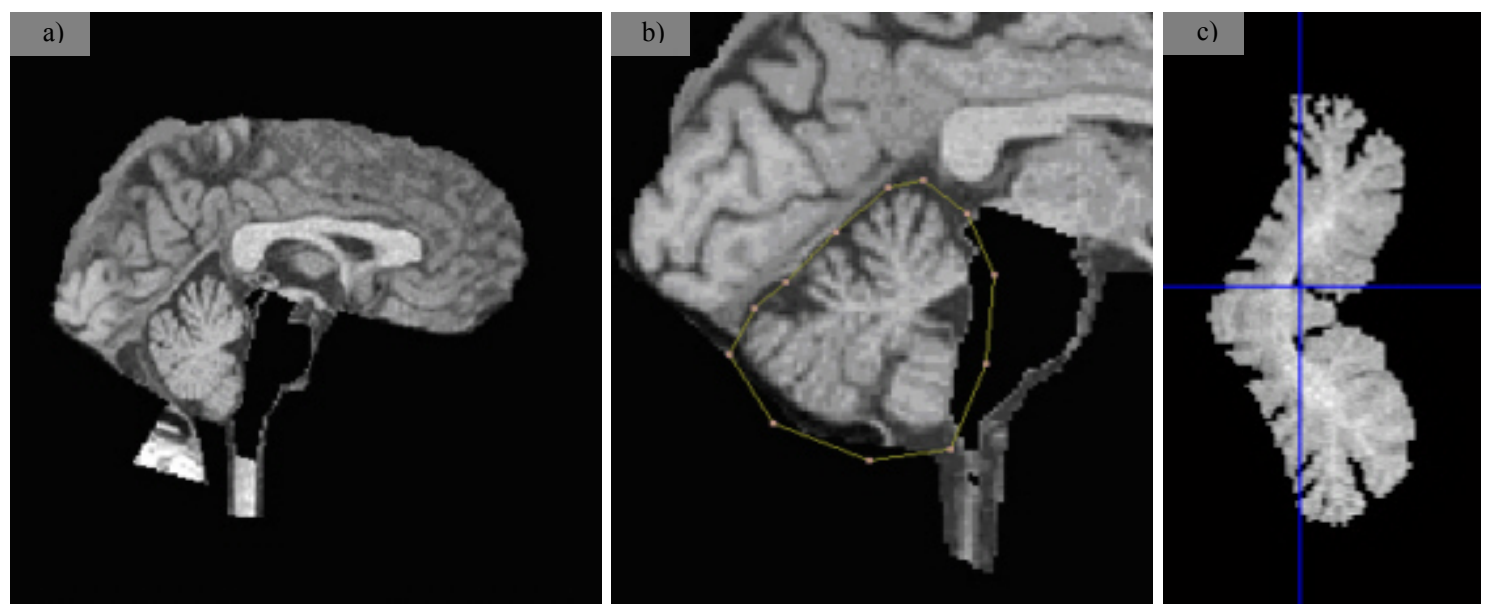

Abb. 3 Manuelle Segmentierung des Zerebellums: a) Subtraktion des Hirnstamms vom Originalvolumen, b) manuelle Segmentierung aufgrund der großen interindividuellen Variationsbreite des Verlaufs vom Tentorium cerebelli, c) Abgrenzung der Hemisphären durch eine paravermale Ebene. 
Eine standardisierte Trennung von Zerebellum und Okzipitallappen war aufgrund der großen morphologischen Variationsbreite des Tentoriums cerebelli nicht möglich. Daher erfolgte die Segmentierung des Zerebellums schichtweise, ausgehend von mediosagittaler Einstellung nach lateral (Abb. 3). Der Vermis wurde von den Hemisphären jeweils durch eine sagittale, paravermale Ebene abgegrenzt.

\section{Basalganglien}

Die Abgrenzung des Putamens nach lateral orientierte sich am Verlauf der Capsula externa, sowie nach medial an der Capsula interna und dem Globus pallidus (Abb. 4a/b). Für die Segmentierung des Nucleus caudatus (Abb. 4c) diente das Vorderhorn des Seitenventrikels als Grenze nach ventral und medial. Die Capsula interna bildete die dorso-laterale Abgrenzung.

Der Globus pallidus war aufgrund eines unzureichenden Gewebekontrastes schwer von den umliegenden Strukturen abzugrenzen und wurde deshalb nicht segmentiert.
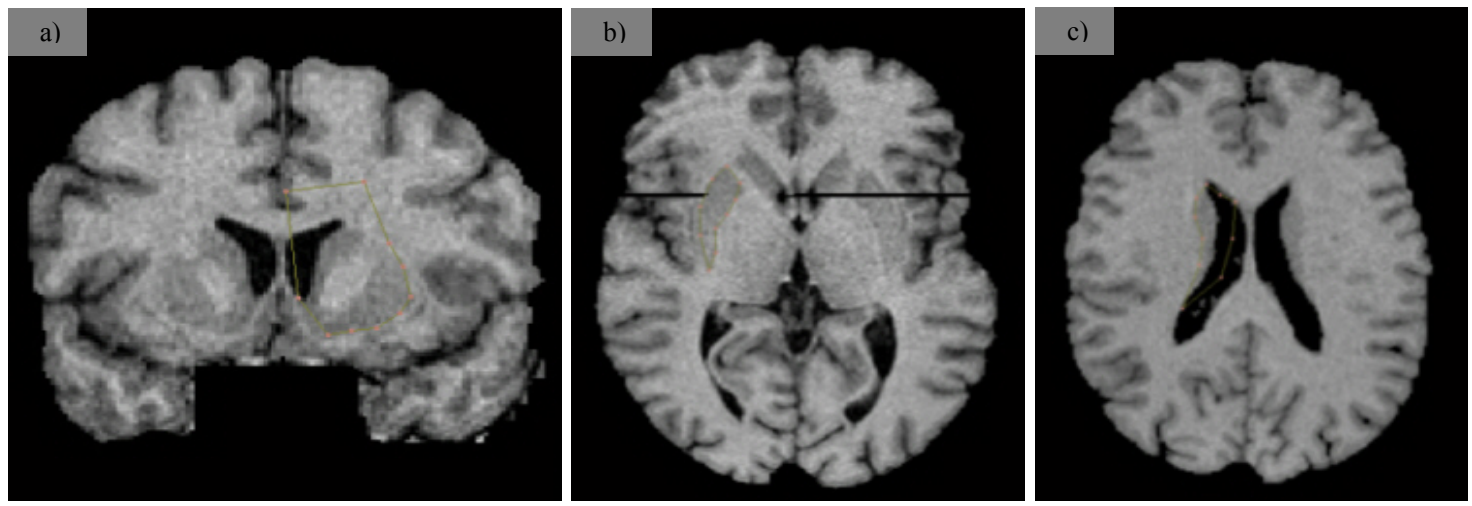

Abb. 4 Manuelle Segmentierung der Basalganglien: a) Markierung der kaudalen Begrenzung des Putamens auf einem Slice in koronarer Schnittführung, b) Segmentierung des Putamens in axialer Ansicht mit Hilfe der zuvor erstellten Markierung als kaudale Abgrenzung, c) Segmentierung des Nucleus caudatus in axialer Ansicht. 


\section{Großhirn}

Nach Subtraktion der bereits segmentierten Basalganglien wurde das Großhirn in eine linke und rechte Hemisphäre zerlegt. Unter Berücksichtigung anatomischer Landmarken (Commissura anterior und posterior) und der Ausdehnung der Hemisphären erfolgte anschließend die Anpassung an das Raster von Talairach (Talairach und Tournoux 1988) (Abb. 5). Dem variablen Verlauf des Sulcus centralis und lateralis wurde durch manuelle Nachbearbeitung Rechnung getragen. Unter Berücksichtigung des Talairachschen Rasters wurden je Hemisphäre vier Körper (Frontal-, Temporal-, Parietal- und Okzipitallappen) erzeugt. Unter Zuhilfenahme eines Grenzwertes konnten zuletzt die Großhirnlappen in weiße und graue Substanz unterteilt werden.
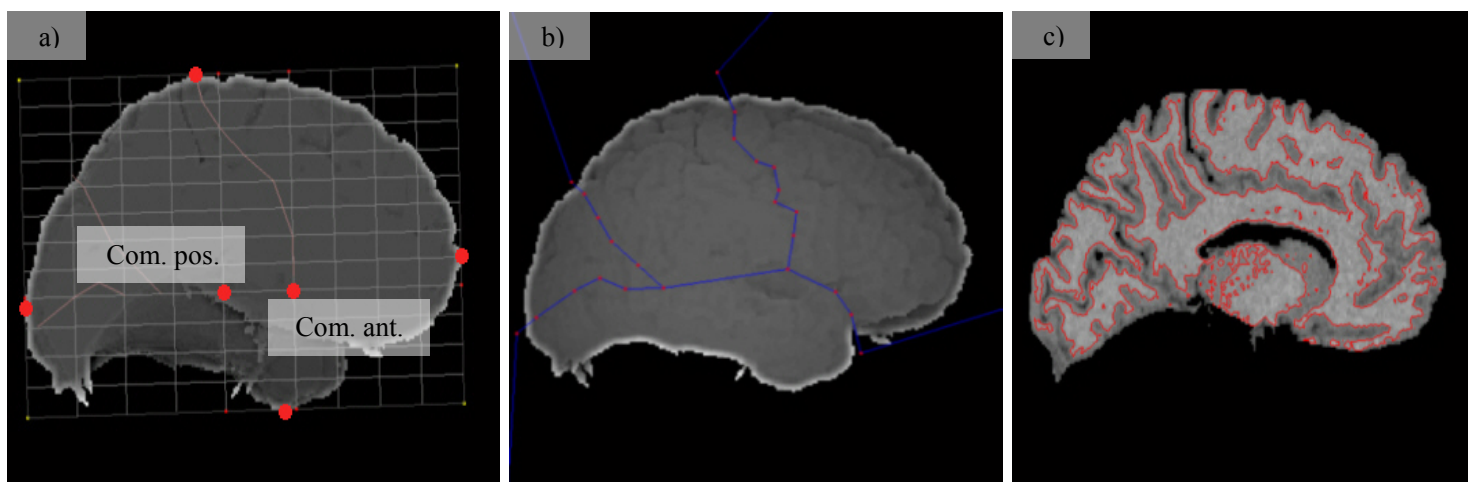

Abb. 5 Segmentierung des Großhirns: a) Definition der anatomischen Landmarken: Commissura anterior und posterior, sowie die kranio-kaudale und anterior-posteriore Ausdehnung des Kortex, b) Segmentierung der Hemisphären unter Zuhilfenahme des Rasters von Talairach, c) Grenzwertbestimmung für die Unterteilung in graue und weiße Substanz.

\section{Totales intrakranielles Volumen}

Das totale intrakranielle Volumen (TICV) wurde unter Berücksichtigung des Verlaufs der Dura mater sowie unter Zuhilfenahme einer 1-zu-10-Stichprobenstrategie (Eritaia et al. 2000) abgeschätzt. 


\subsubsection{Bestimmung des Grenzwerts}

Nach manueller Segmentierung der ROIs wurde eine Grauwertanalyse durchgeführt. Dabei wurden die Voxel (Bildpunkte mit definierter Kantenlänge und Tiefenausdehnung) der ROI sowohl quantitativ als auch qualitativ betrachtet. Während die Quantität für die spätere Volumenbestimmung von Relevanz war, diente die Qualität (Grauwertindex) der Voxel der Gewebedifferenzierung (Abb. 6). Vorausgesetzt wurde hierbei, dass Strukturen ähnlicher Voxelintensität dem gleichen Gewebetyp entsprachen.

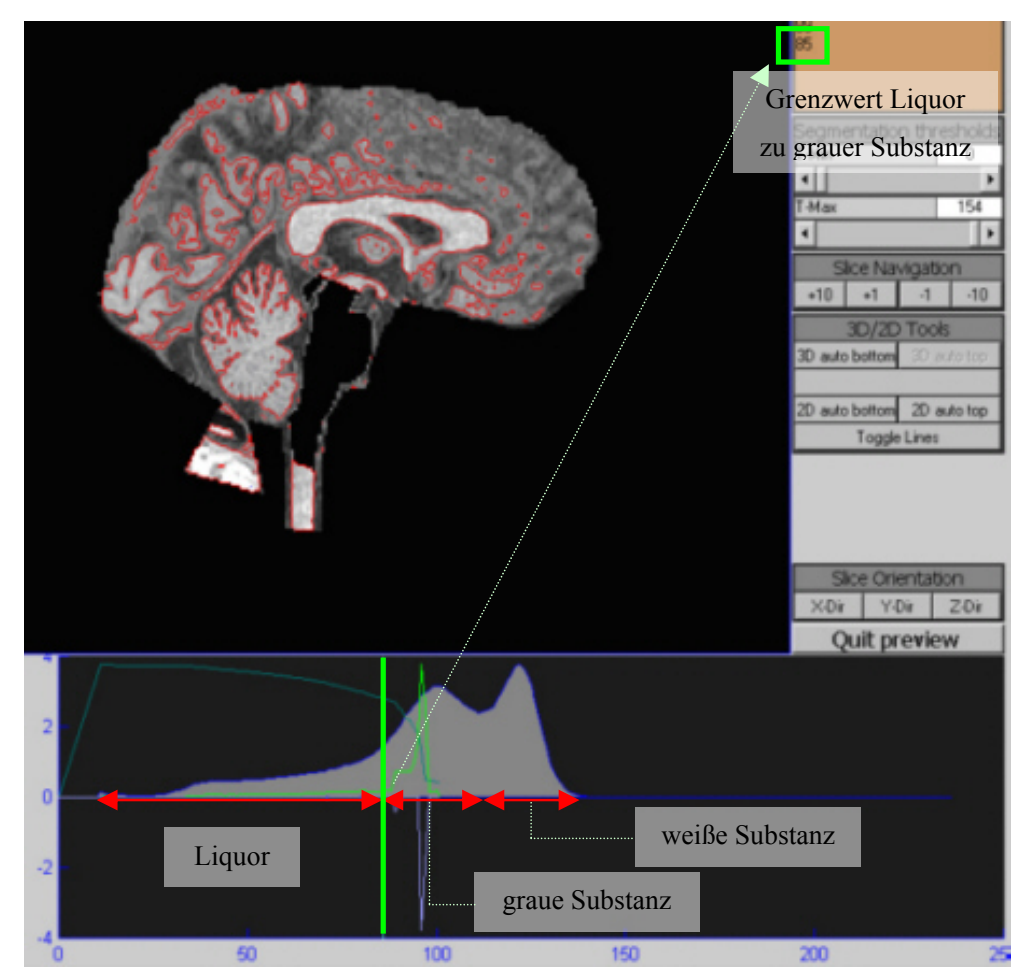

Abb. 6 Die Bestimmung des Grenzwertes am Beispiel der manuellen Segmentierung des Zerebellums. Die Grauwertanalyse (unten) zeigt sowohl die Qualität (Abszisse), als auch die Quantität (Ordinate) der Voxel. Anhand des Kurvenverlaufs können die drei Gewebetypen (Liquor, graue und weiße Substanz) unterschieden werden.

Als threshold (Grenzwert) wurde jeweils der Intensitätswert gewählt, der auf der Abszisse die untere Grenze der zu analysierenden Grauwerte bildete. Für die anschließende Segmentierung waren nur die Grauwerte oberhalb des Grenzwertes relevant. Die Effektivität dieser Volumetrietechnik war somit abhängig von dem jeweiligen Gewebekontrast zwischen der ROI und den angrenzenden Strukturen. 


\subsubsection{Automatischer Region-growing Algorithmus}

Die semiautomatische Segmentierung wurde mit Hilfe eines automatischen, in drei Dimensionen arbeitenden Region-growing Algorithmus (3D-RG) abgeschlossen. Ausgehend von einem Ursprungsvoxel, dem so genannten Saatpunkt, wurden diejenigen Voxel zum Volumen dazugehörig betrachtet, die zum einen innerhalb der zuvor definierten ROI lagen und zum anderen einen Intensitätswert oberhalb des bereits ermittelten Grenzwertes aufwiesen. Resultat des 3D-RG war eine bestimmte Voxelanzahl.

\subsubsection{Volumenberechnung}

Im Anschluss an die Segmentierung konnte das Volumen der Struktur entsprechend der Voxelanzahl berechnet werden. Da es sich teilweise um anisotrope Voxel (Voxel mit unterschiedlicher Kantenausdehnung) handelte, musste dies bei der Volumenberechnung berücksichtigt werden. Um der interindividuellen Varianz der Kopfgrößen Rechnung zu tragen, wurden die berechneten Volumina normalisiert, d.h. sie wurden jeweils durch das TICV dividiert (Schulz et al. 1999; Whitwell et al. 2001; Gröschel et al. 2004).

\subsection{Klinische Parameter}

Die Erkrankungsdauer wurde definiert als der Zeitraum zwischen dem erstmaligen Auftreten einer Störung der Koordination der Bewegungsabläufe und dem Zeitpunkt der MRT-Datenaufnahme.

Um den Schweregrad der Ataxie zu quantifizieren wurde SARA verwendet (SchmitzHübsch et al. 2006b). Diese Skala besteht aus acht Aufgaben und reicht von Null (= keine Ataxie) bis 40 (=schwerste vorstellbare Ausprägung von Ataxie). Die acht Items beschreiben Symptome, die besonders charakteristisch für eine Ataxie sind: 1) Gang ( 0 bis 8 Punkte), 2) Stand (0 bis 6 Punkte), 3) Sitzen (0 bis 4 Punkte), 4) Sprachstörung (0 bis 6 Punkte), 5) Fingerverfolgen (0 bis 4 Punkte), 6) Finger-NaseVersuch (0 bis 4 Punkte), 7) antagonistische Handbewegungen (Pronation / Supination) (0 bis 4 Punkte), 8) Knie-Hacke-Versuch (0 bis 4 Punkte). 


\subsection{Repeatlängen}

Die Repeatlängen des expandierten und des normalen Allels der betroffenen Gene der Studienteilnehmer wurden in der Abteilung Humangenetik der Universität Tübingen bestimmt. Für zehn Patienten waren keine DNA-Proben vorhanden. Mit Hilfe der Patientenakten konnte in sechs Fällen Informationen über die Repeatlängen gewonnen werden. Für die verbleibenden vier Patienten fehlten entsprechende Angaben.

\subsection{Datenanalyse}

Die statistische Analyse erfolgte auf einem Personalcomputer mittels SPSS (Version 15.0) für Windows. Die Daten werden als Mittelwert \pm Standardfehler angegeben.

Für den statistischen Vergleich von Gruppenunterschieden wurde eine Varianzanalyse (ANOVA) unter Berücksichtigung der Kofaktoren Patientenalter und Erkrankungsdauer mit anschließendem Post-Hoc-Test (Fisher LSD) durchgeführt. Die ANOVA quantifiziert die Auswirkung der Gruppenzugehörigkeit (= unabhängige Variable) auf die Volumina der einzelnen anatomischen Strukturen (= abhängige Variable) mit Hilfe des empirischen F-Wertes. Der Vergleich mit dem theoretischen F-Wert entscheidet dann über das Annehmen oder Verwerfen der Nullhypothese. Da die F- und p-Werte der ANOVA von der Gruppengröße und der Varianz der Daten abhängen, ermöglichen sie keine direkte Aussage über das Ausmaß der Gruppenunterschiede. Um ein besseres Maß für die Effektstärke der Mittelwertunterschiede zu erhalten, wurde das Cohens $d$ herangezogen. Die Effektstärke Cohens $d$ ist unabhängig von der Gruppengröße und erlaubt somit den direkten Vergleich der Gruppen untereinander. Die Effektstärke kann auch interpretiert werden als Ausdruck des prozentualen Nichtüberlappens der Gruppenpunktzahl (Cohen 1988).

Um den Zusammenhang zwischen den regionalen Hirnvolumina und dem Ausmaß der klinischen Beeinträchtigung, der Erkrankungsdauer und der Länge des expandierten Allels zu untersuchen, wurde der Pearson'sche Korrelationskoeffizienten verwendet. Dieser Koeffizient ist ein dimensionsloser Index (Wertebereich: $-1 \leq \mathrm{r} \leq+1$ ), der die lineare Abhängigkeit zweier Variablen überprüft. Da die Absicht darin bestand, Korrelationen neu zu entdecken und nicht zu bestätigen, wurden diese Korrelationsberech- 
nungen nicht für multiples Überprüfen korrigiert. Mit Hilfe einer multiplen Regressionsanalyse konnte für jeden Genotyp gezeigt werden, welche anatomischen Strukturen einen signifikanten Beitrag für die Varianz der klinischen Dysfunktion (SARA) leisteten.

Die Prognose der Gruppenzugehörigkeit der einzelnen Elemente (Klassifizierung) wurde durch die abschließende schrittweise Diskriminanzanalyse ermöglicht. Sie gab Aufschluss, welche Variable (anatomische Struktur) sich besonders zur Unterscheidung der Gruppen (Kontrollpersonen, SCA1, SCA3 und SCA6) eignet. Das mathematische Äquivalent dieser Fragestellung ist die so genannte kanonische Diskriminanzfunktion (Trennfunktion). Mit ihrer Hilfe ist eine optimale Trennung zwischen den Gruppen sowie die Prüfung der diskriminatorischen Bedeutung der einzelnen Merkmalsvariablen möglich. 


\section{$3 \quad$ Ergebnisse}

\subsection{Charakteristika der Kontrollgruppe und des Patientenkollektivs}

Insgesamt wurden für die Multizenterstudie 31 Kontrollpersonen sowie 48 SCA1-, 24 SCA3- und 10 SCA6-Patienten rekrutiert (Tabelle 3). Das Alter der Patienten $[F(3 ; 109)=5,31 ; p<0,002]$ zum Zeitpunkt der Untersuchung war für Patienten mit SCA1 (Alter: 44,3 $\pm 11,9$ Jahre) und Patienten mit SCA3 (Alter: 47,3 $\pm 11,4$ Jahre) ähnlich. Für Patienten mit SCA6 (Alter: 61,9 $\pm 10,2$ Jahre) war es signifikant höher. Im Hinblick auf die Erkrankungsdauer $[\mathrm{F}(2 ; 79)=3,98 ; \mathrm{p}=0,023]$ gab es keine signifikanten Unterschiede

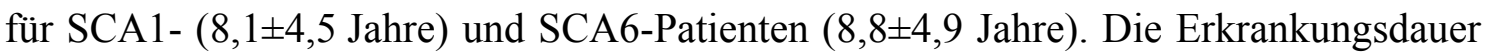
für SCA3-Patienten (11,7 $\pm 6,0$ Jahre) hingegen war signifikant länger.

Tabelle 3 Demographische und klinische Charakteristika der Kontrollgruppe und des Patientenkollektivs; die Daten werden als Mittelwert \pm Standardfehler angegeben; die statistische Auswertung erfolgte mit Hilfe einer Varianzanalyse (ANOVA) und anschließendem Post-Hoc-Test (Fisher LSD); Signifikanzen: * $\mathrm{p}<0,05$ im Vergleich mit Kontrollpersonen, SCA1 und SCA3, ${ }^{*} \mathrm{p}<0,05$ im Vergleich mit SCA1.

\begin{tabular}{|c|c|c|c|c|}
\hline \multirow{2}{*}{ Charakteristika } & \multirow{2}{*}{$\begin{array}{c}\text { Kontroll- } \\
\text { personen }\end{array}$} & SCA1 & SCA3 & SCA6 \\
\cline { 3 - 5 } & $31(14 / 17)$ & $48(31 / 17)$ & $24(11 / 13)$ & $10(9 / 1)$ \\
\hline Patientenzahl n (m/w) & & & $47,3 \pm 11,4$ & $61,9 \pm 10,2^{*}$ \\
\hline Patientenalter & $49,8 \pm 16,2$ & $44,3 \pm 11,9$ & $(29-71)$ & $(43-72)$ \\
\hline (Spannweite) & $(16-76)$ & $(18-72)$ & $11,7 \pm 6,0^{\#}$ & $8,8 \pm 4,9$ \\
\hline Erkrankungsdauer & - & $8,1 \pm 4,5$ & $35,6 \pm 9,7$ & $53,1 \pm 10,3$ \\
\hline Krankheitsbeginn & - & $36,2 \pm 10,8$ & $(19-59)$ & $(37-66)$ \\
\hline (Spannweite) & - & $47,7 \pm 5,8$ & $69,2 \pm 3,4$ & $22,3 \pm 0,5$ \\
\hline CAG-Repeatlänge & - & $(39-66)$ & $(61-76)$ & $(22-23)$ \\
\hline (Spannweite) & - & $12,0 \pm 5,2$ & $12,0 \pm 5,7$ & $12,7 \pm 6,0$ \\
\hline SARA & & & & \\
\hline
\end{tabular}

Die mittlere CAG-Repeatlänge betrug 47,7 für SCA1 (Spannweite: 39-66), 69,2 für SCA3 (Spannweite: 61-76) und 22,3 für SCA6 (Spannweite: 22-23). Der klinische Schweregrad der Erkrankung, der durch SARA erfasst wurde, war für alle Gruppen ähnlich. 


\subsection{Volumetrie}

Das TICV von Männern war signifikant größer als das von Frauen [65 männliche Personen: $1548 \pm 141 \mathrm{~cm}^{3} ; 48$ weibliche Personen: $1371 \pm 95 \mathrm{~cm}^{3} ; \mathrm{F}(1 ; 111)=57,16$; $\mathrm{p}<0,0001]$. Zwischen dem Patientenalter und dem TICV bestanden keine signifikanten Zusammenhänge. Die einzelnen Gruppen unterschieden sich im Hinblick auf das TICV nicht (Tabelle 4).

Tabelle 4 Normalisierte Volumina der ROIs von Kontrollgruppe und Patientenkollektiv, korrigiert für Patientenalter und Erkrankungsdauer; die statistische Auswertung erfolgte mit Hilfe einer Varianzanalyse (ANOVA) und anschließendem Post-Hoc-Test (Fisher LSD); die Signifikanzen sind für diejenigen Gruppen angegeben, bei denen im Vergleich mit einer anderen Gruppen eine signifikante Reduzierung des Volumens bestand: * $\mathrm{p}<0,05$ im Vergleich mit Kontrollpersonen, ${ }^{\S} \mathrm{p}<0,05$ im Vergleich mit SCA1, ${ }^{\#} \mathrm{p}<0,05 \mathrm{im}$ Vergleich mit SCA3, ${ }^{\circ} \mathrm{p}<0,05$ im Vergleich mit SCA6.

\begin{tabular}{|l|c|c|c|c|c|}
\hline ROI (in \% von TICV) & Kontrollpersonen & SCA1 & SCA3 & SCA6 & F(3,109) \\
\hline TICV $\left(\mathrm{cm}^{3}\right)$ & $1451,4 \pm 125,3$ & $1464,3 \pm 150,7$ & $1468,4 \pm 158,8$ & $1592,8 \pm 177,2$ & 2,5 \\
\hline Hirnstamm & $2,19 \pm 0,19$ & $1,45 \pm 0,17^{* \circ}$ & $1,51 \pm 0,23^{* \circ}$ & $2,02 \pm 0,16^{*}$ & $\mathbf{1 1 3 , 5}$ \\
Mesenzephalon & $0,35 \pm 0,07$ & $0,32 \pm 0,05$ & $0,29 \pm 0,05^{\S \circ}$ & $0,35 \pm 0,06$ & $\mathbf{4 , 3}$ \\
Pons & $1,37 \pm 0,18$ & $0,78 \pm 0,13^{*} \circ$ & $0,87 \pm 0,20^{* \circ}$ & $1,25 \pm 0,15^{*}$ & $\mathbf{9 8 , 4}$ \\
Medulla oblongata & $0,47 \pm 0,08$ & $0,33 \pm 0,06^{* \circ}$ & $0,32 \pm 0,06^{* \circ}$ & $0,44 \pm 0,07$ & $\mathbf{4 1 , 8}$ \\
\hline Zerebellum & $8,58 \pm 0,75$ & $6,72 \pm 0,7 *^{* \#}$ & $7,42 \pm 0,66^{*}$ & $6,64 \pm 0,67^{*}$ & $\mathbf{4 3 , 6}$ \\
Hemisphären & $7,67 \pm 0,63$ & $6,23 \pm 0,74^{* \#}$ & $6,54 \pm 0,60^{*}$ & $6,38 \pm 0,61^{*}$ & $\mathbf{3 0 , 3}$ \\
Vermis & $0,91 \pm 0,27$ & $0,44 \pm 0,10^{*}$ & $0,46 \pm 0,11^{*}$ & $0,36 \pm 0,07 *$ & $\mathbf{6 1 , 4}$ \\
\hline Putamen + Nucleus caudatus & $1,01 \pm 0,10$ & $0,83 \pm 0,11^{* \circ}$ & $0,87 \pm 0,11^{* \circ}$ & $0,99 \pm 0,06$ & $\mathbf{2 0 , 0}$ \\
Putamen & $0,52 \pm 0,07$ & $0,41 \pm 0,07^{*} \circ$ & $0,45 \pm 0,07^{* \circ}$ & $0,52 \pm 0,04$ & $\mathbf{1 8 , 0}$ \\
Nucleus caudatus & $0,49 \pm 0,06$ & $0,42 \pm 0,05^{* \circ}$ & $0,42 \pm 0,05^{* \circ}$ & $0,46 \pm 0,03$ & $\mathbf{1 3 , 4}$ \\
\hline Hemisphären & $69,25 \pm 3,56$ & $69,19 \pm 4,69$ & $68,43 \pm 3,55$ & $70,40 \pm 3,24$ & 0,6 \\
Frontallappen & $22,56 \pm 1,38$ & $22,00 \pm 1,73$ & $22,43 \pm 1,54$ & $22,73 \pm 0,61$ & 1,4 \\
Temporallappen & $18,60 \pm 1,79$ & $16,81 \pm 1,64^{* \circ}$ & $16,71 \pm 1,62^{* \circ}$ & $18,64 \pm 2,13$ & $\mathbf{9 , 9}$ \\
Parietallappen & $23,07 \pm 1,90^{\S \circ}$ & $24,54 \pm 1,82$ & $23,78 \pm 1,50$ & $24,95 \pm 1,68$ & $\mathbf{5 , 4}$ \\
Okzipitallappen & $5,01 \pm 0,56$ & $5,23 \pm 0,48$ & $5,22 \pm 0,38$ & $5,49 \pm 0,59$ & 2,6 \\
\hline
\end{tabular}

Für die graphische Darstellung der statistischen Kenngrößen wurde der Box-andWhisker-Plot, der den direkten Vergleich von Stichproben ermöglicht, gewählt. Die Box wird jeweils begrenzt von den beiden Quartilen. Innerhalb der Box ist der Median (Quantil) eingezeichnet. Die Länge der Box entspricht dem Interquartilsabstand (interquartile range, IQR). Die Länge der Schnurrhaare (engl. whiskers) beträgt maximal 1,5*IQR. Werte im Bereich von 1,5*IQR $<\mathrm{x}<3 * \mathrm{IQR}$ werden als milde (Symbol: + ) und diejenigen mit $\mathrm{x}>3 * \mathrm{IQR}$ als extreme (Symbol: $\Delta$ ) Ausreißer bezeichnet. 
Der Trend zu höheren Volumina bei SCA6 ist bedingt durch einen höheren prozentualen Anteil männlicher Patienten in dieser Gruppe (Abb. 7). Um diese Unterschiede und die interindividuelle Varianz der Kopfgrößen auszugleichen, wurden die einzelnen ROIs

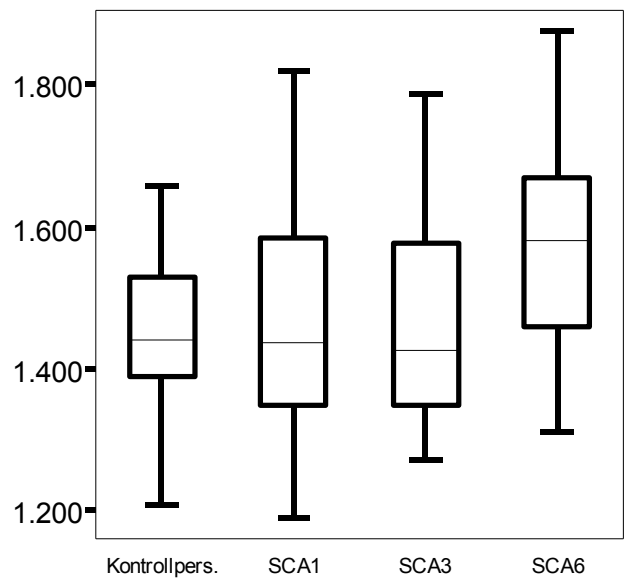
mit Hilfe des TICV normalisiert (Tabelle 4). Da das Patientenalter und die Erkrankungsdauer zwischen den Gruppen unterschiedlich waren, wurden die Ausgangsdaten außerdem für das Patientenalter und die Erkrankungsdauer korrigiert (Tabelle 4).

Abb. 7 TICV $\left[\mathrm{cm}^{3}\right]$ (Ordinate) der Kontrollpersonen und des Patientenkollektivs (Abszisse).

Aufgrund der unterschiedlichen Größe der einzelnen Gruppen war es nicht möglich, das Ausmaß der Gruppenunterschiede direkt miteinander zu vergleichen. Um dieser Tatsache Rechnung zu tragen, wurde die Effektstärke Cohens $d$ berechnet. Diese statistische Maßzahl ist unabhängig von der Gruppengröße und ermöglicht den direkten Vergleich der Gruppen untereinander. Abb. 8 zeigt, dass die Gruppenunterschiede für die Strukturen des Hirnstamms am größten sind.

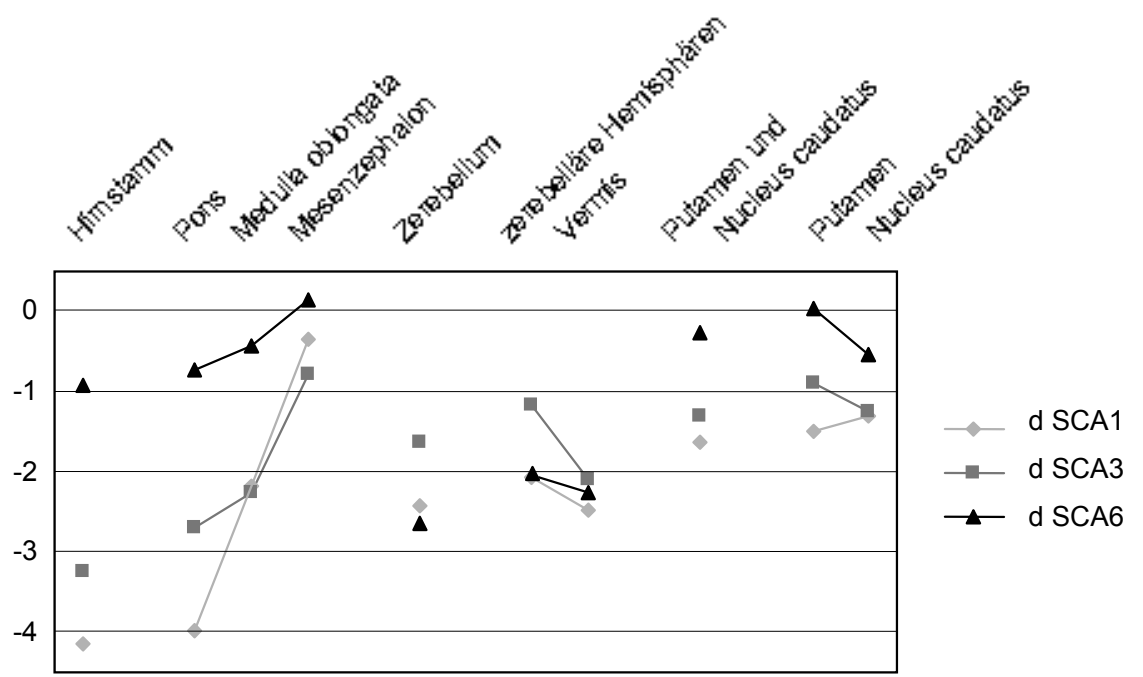

Abb. 8 Die Effektstärke Cohens $d$ (Ordinate) beschreibt das Ausmaß der Atrophie einzelner Strukturen (Abszisse). Diese Größe ist unabhängig von der Gruppengröße und ermöglicht den direkten Vergleich der einzelnen SCA-Genotypen. 
Das durchschnittliche, normalisierte, nach Patientenalter und Erkrankungsdauer korrigierte Hirnstammvolumen der Kontrollgruppe betrug 2,19 $\pm 0,19 \%$ des TICV (Tabelle 4). Unter Zuhilfenahme der Effektstärke Cohens $d$ konnte gezeigt werden, dass die Gruppenunterschiede für die Strukturen des Hirnstamms am größten waren (Abb. 8). Bei SCA1 war die Atrophie des Pons im Vergleich zu SCA3 und SCA6 wesentlich stärker ausgeprägt (Abb. 9). Außerdem zeigten SCA1 und SCA3 im Vergleich zu den Kontrollpersonen signifikante Unterschiede für den gesamten Hirnstamm und die Medulla oblongata. Die Atrophie bei SCA6 hingegen war wesentlich schwächer ausgeprägt.

a) Hirnstamm

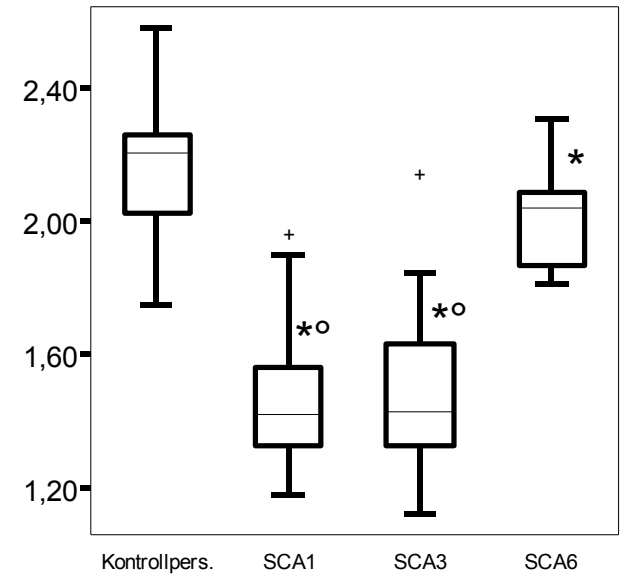

c) Pons

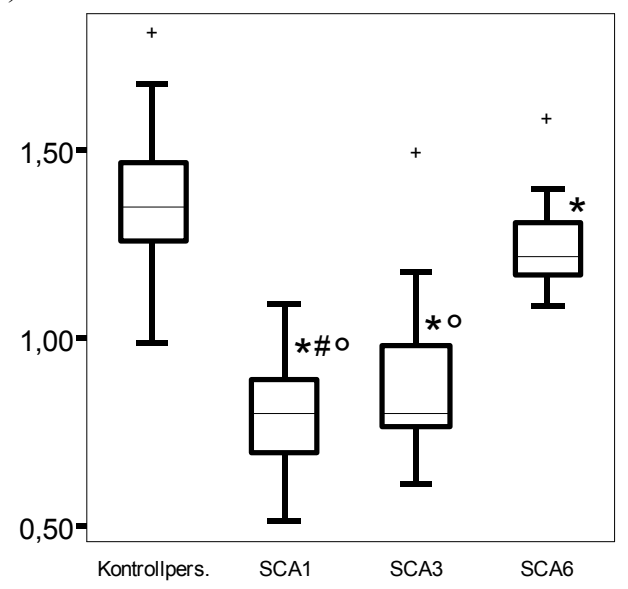

b) Mesenzephalon

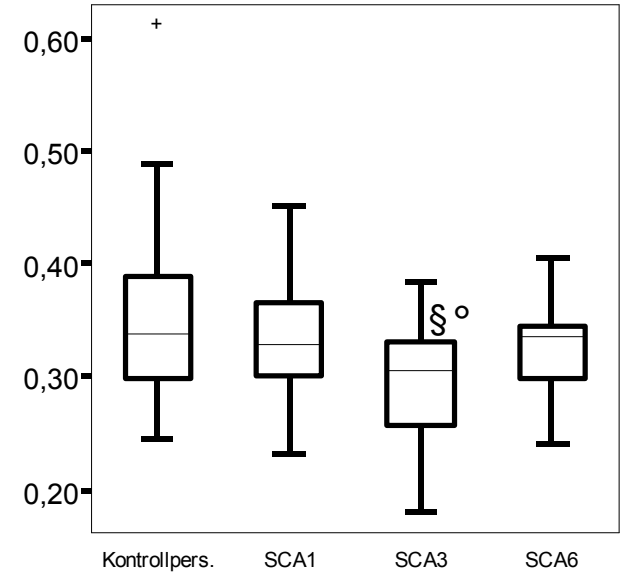

d) Medulla oblongata

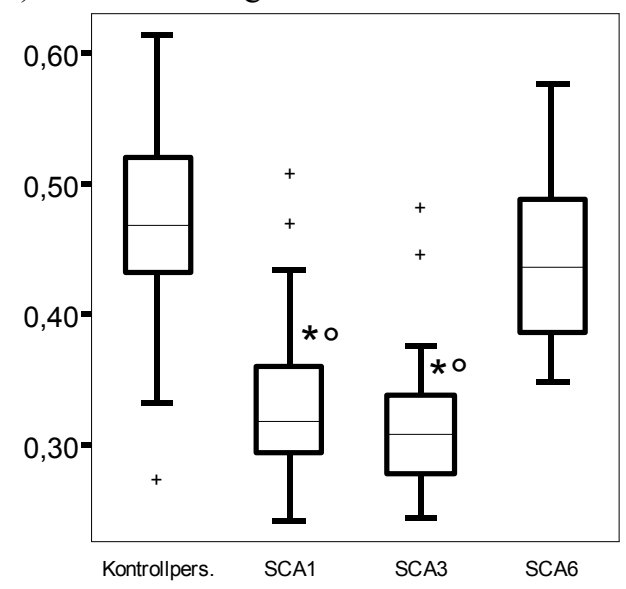

Abb. 9 Normalisierte Volumina [\% vom TICV] (Ordinate) der Kontrollpersonen und des Patientenkollektivs (Abszisse) von a) Hirnstamm, b) Mesenzephalon, c) Pons und d) Medulla oblongata. Signifikanzen: * $\mathrm{p}<0,05$ im Vergleich mit Kontrollpersonen, ${ }^{\S} \mathrm{p}<0,05 \mathrm{im}$ Vergleich mit SCA1, ${ }^{\#} \mathrm{p}<0,05$ im Vergleich mit SCA3, ${ }^{\circ} \mathrm{p}<0,05$ im Vergleich mit SCA6. + : milde Ausreißer. 
Das durchschnittliche, normalisierte, nach Patientenalter und Erkrankungsdauer korri-

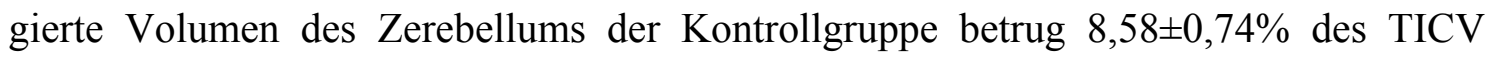
(Tabelle 4). Im Vergleich zu den Kontrollpersonen zeigten SCA1, SCA3 und SCA6 atrophische Veränderungen des Zerebellums. Insbesondere die Atrophie des Vermis war bei allen drei Gruppen stark ausgeprägt (Abb. 10).

a) Zerebellum

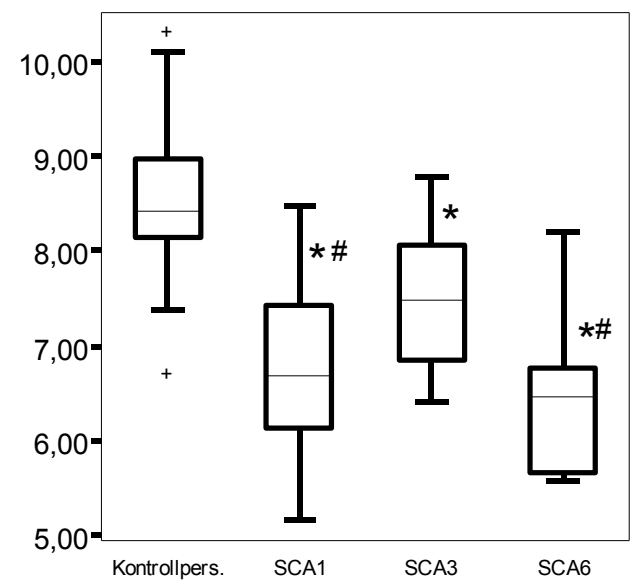

b) zerebelläre Hemisphären

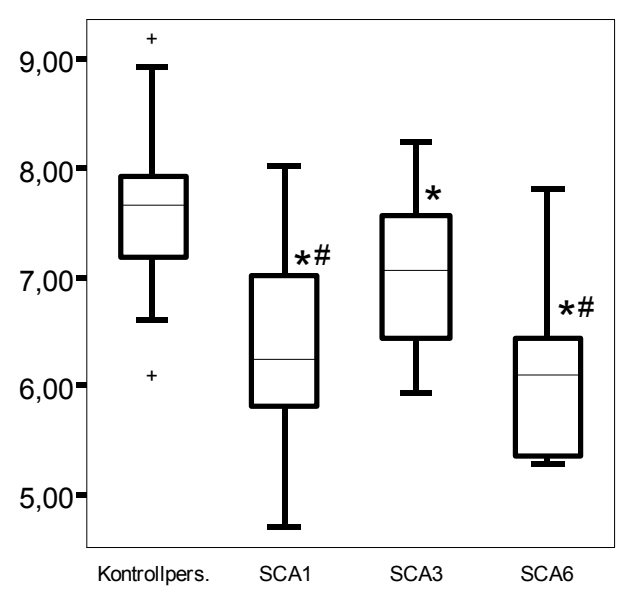

c) Vermis

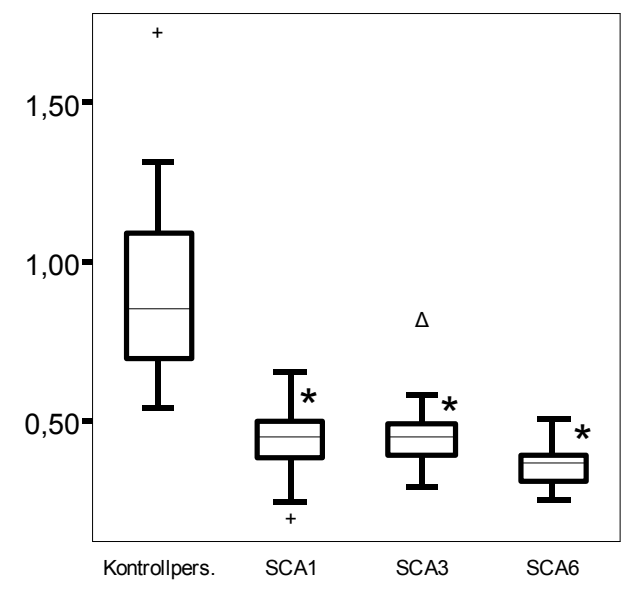

Abb. 10 Normalisierte Volumina [\% vom TICV] (Ordinate) der Kontrollpersonen und des Patientenkollektivs (Abszisse) von a) Zerebellum, b) zerebelläre Hemisphären, c) Vermis. Signifikanzen: * $\mathrm{p}<0,05$ im Vergleich mit Kontrollpersonen, ${ }^{*} \mathrm{p}<0,05$ im Vergleich mit SCA3. + : milde Ausreißer, $\Delta$ : extreme Ausreißer. 
Das durchschnittliche, normalisierte, nach Patientenalter und Erkrankungsdauer korrigierte Volumen von Putamen und Nucleus caudatus der Kontrollgruppe betrug $1,01 \pm 0,10 \%$ des TICV (Tabelle 4). SCA1 und SCA3 unterschieden sich im Hinblick auf Atrophien signifikant von der Kontrollgruppe. Bei SCA6 hingegen war das Volumen von Putamen und Nucleus caudatus nicht signifikant reduziert (Abb. 11).

a) Putamen und Nucleus caudatus

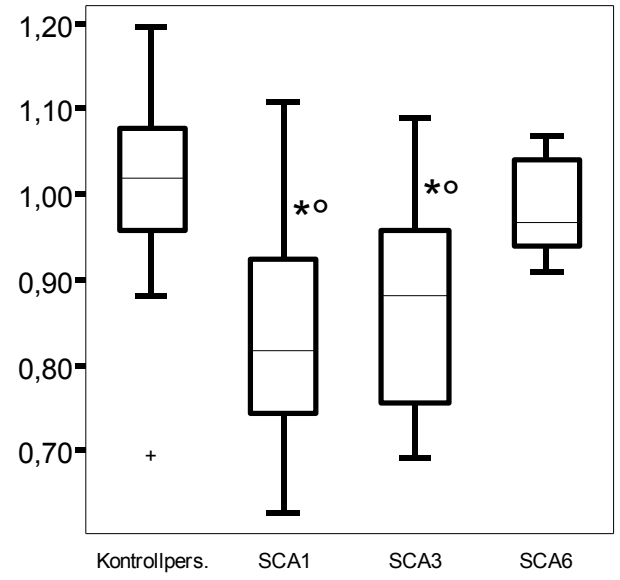

b) Putamen

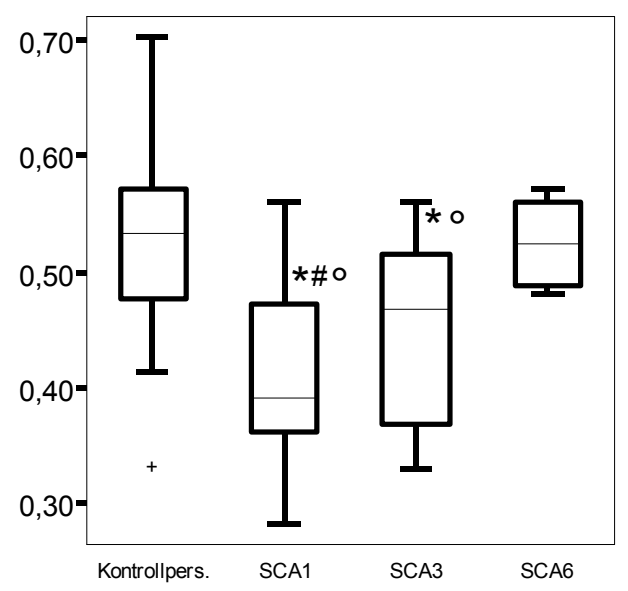

c) Nucleus caudatus

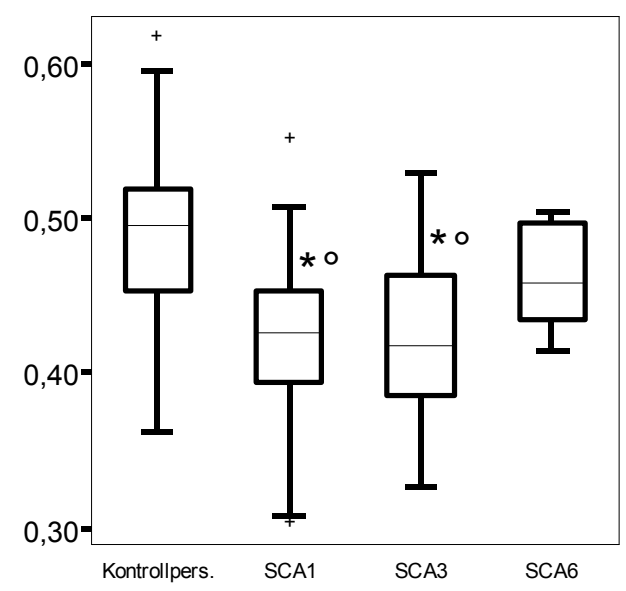

Abb. 11 Normalisierte Volumina [\% vom TICV] (Ordinate) der Kontrollpersonen und des Patientenkollektivs (Abszisse) von a) Putamen und Nucleus caudatus, b) Putamen und c) Nucleus caudatus. Signifikanzen: ${ }^{*} \mathrm{p}<0,05$ im Vergleich mit Kontrollpersonen, ${ }^{*} \mathrm{p}<0,05 \mathrm{im}$ Vergleich mit SCA3, ${ }^{\circ} \mathrm{p}<0,05$ im Vergleich mit SCA6. + : milde Ausreißer. 
Das durchschnittliche, normalisierte, nach Patientenalter und Erkrankungsdauer korrigierte Volumen der zerebralen Hemisphären der Kontrollgruppe betrug 69,25 $\pm 3,56 \%$ des TICV (Tabelle 4). Es konnten keine systematischen, den gesamten Kortex betreffenden atrophischen Veränderungen nachgewiesen werden (Abb. 12). Mit Hilfe des Talairachschen Rasters und unter Berücksichtigung anatomischer Landmarken wurden bei SCA1 und SCA3 Atrophien des Temporallappens entdeckt. Allerdings zeigten dieselben Patienten zur gleichen Zeit ein Anwachsen des Parietallappens. Dieser Zusammenhang deutet auf einen systematischen Fehler des Segmentierungsalgorithmus hin und wird daher als Artefakt interpretiert.

a) Frontallapen

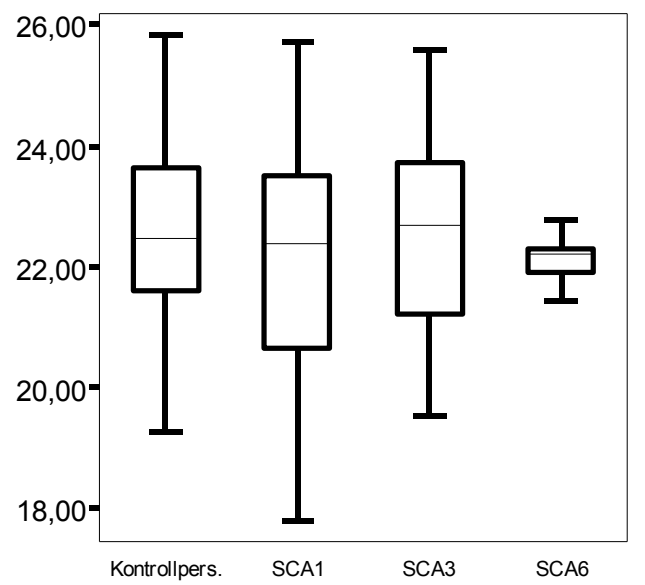

c) Parietallappen

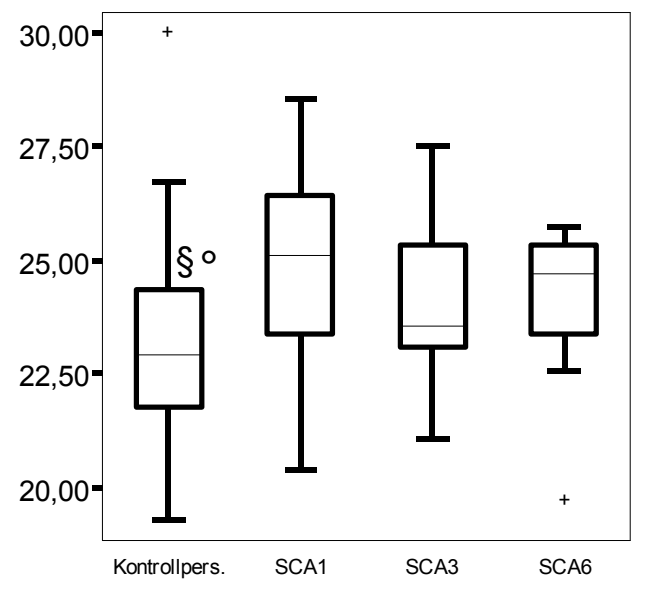

b) Temporallappen

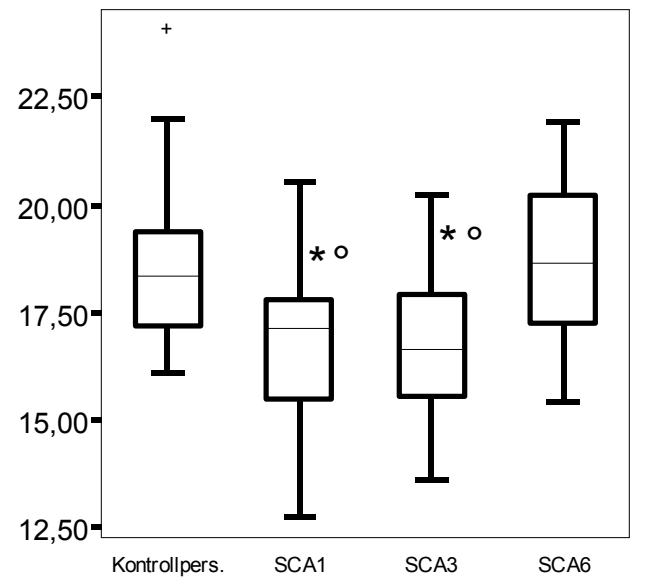

d) Okzipitallappen

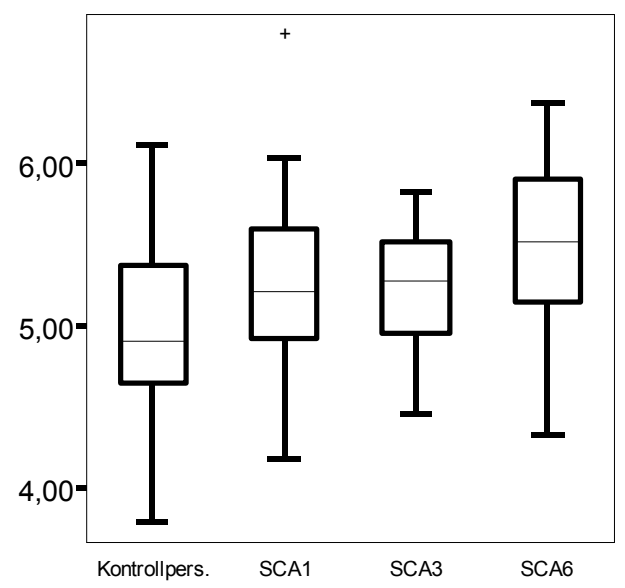

Abb. 12 Normalisierte Volumina [\% vom TICV] (Ordinate) der Kontrollpersonen und des Patientenkollektivs (Abszisse) von a) Frontallappen, b) Temporallappen, c) Parietallappen und d) Okzipitallappen. Signifikanzen: ${ }^{*} \mathrm{p}<0,05$ im Vergleich mit Kontrollpersonen, ${ }^{\S} \mathrm{p}<0,05 \mathrm{im}$ Vergleich mit SCA1, ${ }^{\circ} \mathrm{p}<0,05$ im Vergleich mit SCA6. + : milde Ausreißer. 


\subsection{Korrelationen}

\subsubsection{Volumina, korreliert mit den klinischen Symptomen (SARA)}

Der Schweregrad der Ataxie, der mit Hilfe von SARA gemessen wurde, war für alle

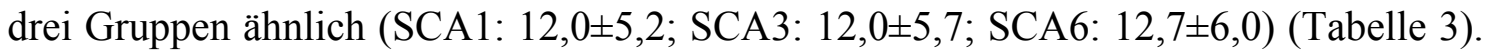
Bei SCA1 korrelierte dieser Wert mit den atrophischen Veränderungen in Hirnstamm und Pons (Tabelle 5). Bei SCA3 bestand für folgende Strukturen ein signifikanter Zusammenhang zwischen SARA und der Atrophie: Hirnstamm, Mesenzephalon, Pons, Medulla oblongata, Zerebellum, zerebelläre Hemisphären und Nucleus caudatus. Bei SCA6 korrelierte SARA mit der Atrophie des Zerebellums und der zerebellären Hemisphären.

Tabelle 5 Korrelation einzelner neuroanatomischer Strukturen mit den klinischen Symptomen (SARA); die statistische Auswertung erfolgte mit Hilfe des Pearson'sche Korrelationskoeffizienten; Signifikanzen: $* \mathrm{p}<0,05 ; * * \mathrm{p}<0,01 ; * * * \mathrm{p}<0,001$.

\begin{tabular}{|c|c|c|c|}
\hline & SARA / SCA1 & SARA / SCA3 & SARA / SCA6 \\
\hline Hirnstamm [\%] & $-0,447^{* *}$ & $-0,677^{* *}$ & $-0,312$ \\
Mesenzephalon [\%] & $-0,061$ & $-0,467 *$ & 0,055 \\
Pons [\%] & $-0,531^{* * *}$ & $-0,560^{* *}$ & $-0,063$ \\
Medulla oblongata [\%] & $-0,011$ & $-0,479 *$ & $-0,625$ \\
\hline Zerebellum [\%] & $-0,187$ & $-0,451^{*}$ & $-0,770^{* *}$ \\
Hemisphären [\%] & $-0,208$ & $-0,459^{*}$ & $-0,751^{*}$ \\
Vermis [\%] & 0,082 & $-0,130$ & $-0,568$ \\
\hline Putamen + Nucleus caudatus [\%] & $-0,141$ & $-0,355$ & $-0,202$ \\
Putamen [\%] & $-0,087$ & $-0,240$ & $-0,009$ \\
Nucleus caudatus [\%] & $-0,189$ & $-0,455^{*}$ & $-0,361$ \\
\hline
\end{tabular}




\subsubsection{Volumina, korreliert mit den CAG-Repeatlängen}

Die CAG-Repeatlänge des expandierten Allels zeigte bei SCA1 und SCA3 einen signifikanten Zusammenhang mit SARA (Tabelle 6). Bei SCA6 hingegen konnte keine Korrelation mit SARA beobachtet werden. Eine inverse Korrelation zwischen der CAGRepeatlänge und dem Ausmaß der Atrophie wurde bei SCA3 für die Strukturen Hirnstamm, Mesenzephalon, Putamen und Nucleus caudatus beobachtet. Bei SCA1 bestand indes nur ein Trend zu einer inversen Korrelation mit der Atrophie des Pons.

Tabelle 6 Korrelation einzelner neuroanatomischer Strukturen mit den CAG-Repeatlängen; die partiellen Korrelationen sind korrigiert für Erkrankungsdauer und Patientenalter; Signifikanzen: ${ }^{+} \mathrm{p}=0,055 ; * \mathrm{p}<0,05 ; * * \mathrm{p}<0,01 ; * * * \mathrm{p}<0,001$.

\begin{tabular}{|l|c|c|c|}
\hline & SCA1 & SCA3 & SCA6 \\
\hline SARA & $0,387 * *$ & $0,642^{* *}$ & $-0,272$ \\
\hline Hirnstamm [\%] & $-0,167$ & $-0,543 * *$ & 0,458 \\
Mesenzephalon [\%] & $-0,011$ & $-0,658^{* * *}$ & $-0,127$ \\
Pons [\%] & $-0,289+$ & $-0,379$ & 0,603 \\
Medulla oblongata [\%] & 0,177 & $-0,280$ & $-0,069$ \\
\hline Zerebellum [\%] & $-0,154$ & $-0,366$ & 0,518 \\
Hemisphären [\%] & $-0,169$ & $-0,320$ & 0,543 \\
Vermis [\%] & 0,047 & $-0,414$ & 0,187 \\
\hline Putamen + Nucleus caudatus [\%] & 0,097 & $-0,533^{*}$ & 0,060 \\
Putamen [\%] & 0,124 & $-0,436 *$ & $-0,217$ \\
Nucleus caudatus [\%] & 0,059 & $-0,551 * *$ & 0,355 \\
\hline
\end{tabular}




\subsubsection{Volumina, korreliert mit der Erkrankungsdauer}

Obwohl die Erkrankungsdauer bei SCA1, SCA3 und SCA6 mit SARA korrelierte, gab es nur wenig signifikante Zusammenhänge zwischen der Atrophie und der Erkrankungsdauer (Tabelle 7). Bei SCA1 korrelierte die Erkrankungsdauer nur mit der Volumenabnahme des Pons. Bei SCA6 bestand beinahe ein signifikanter Zusammenhang mit der Atrophie des Zerebellums und der zerebellären Hemisphären $(p=0,07)$. Bei SCA3 konnten keine Zusammenhänge zwischen Erkrankungsdauer und Atrophie beobachtet werden.

Tabelle 7 Korrelation einzelner neuroanatomischer Strukturen mit der Erkrankungsdauer; die statistische Auswertung erfolgte mit Hilfe des Pearson'sche Korrelationskoeffizienten; Signifikanzen: ${ }^{+} \mathrm{p}=0,07 ; * \mathrm{p}<0,05 ; * * \mathrm{p}<0,01 ; * * * \mathrm{p}<0,001$.

\begin{tabular}{|l|c|c|c|}
\hline & SCA1 & SCA3 & SCA6 \\
\hline SARA & $0,617^{* * *}$ & $0,387^{*}$ & $0,683^{*}$ \\
\hline Hirnstamm [\%] & $-0,130$ & $-0,281$ & $-0,156$ \\
Mesenzephalon [\%] & 0,106 & $-0,297$ & 0,297 \\
Pons [\%] & $-0,311^{*}$ & $-0,248$ & $-0,135$ \\
Medulla oblongata [\%] & 0,262 & $-0,048$ & $-0,296$ \\
\hline Zerebellum [\%] & $-0,058$ & $-0,153$ & $-0,495^{+}$ \\
Hemisphären [\%] & $-0,101$ & $-0,189$ & $-0,499^{+}$ \\
Vermis [\%] & 0,301 & 0,167 & $-0,201$ \\
\hline Putamen + Nucleus caudatus [\%] & $-0,162$ & $-0,269$ & 0,124 \\
Putamen [\%] & $-0,126$ & $-0,273$ & 0,149 \\
Nucleus caudatus [\%] & $-0,178$ & $-0,207$ & 0,056 \\
\hline
\end{tabular}




\subsubsection{Regressionsanalyse für die Varianz von SARA}

Welchen Beitrag die einzelnen neuroanatomischen Strukturen innerhalb einer Gruppe für die Varianz von SARA leisteten, wurde mit Hilfe einer schrittweisen linearen Regressionsanalyse untersucht. Die statistischen Zusammenhänge wurden bei $p_{\text {in }}=0,10$ und $\mathrm{p}_{\text {out }}=0,25$ erreicht.

Bei SCA1 erklärte das Volumen des Pons zu $28 \%$ die Varianz von SARA $[F(1 ; 46)=18,0 ; p<0,001]$. Keine weiteren Strukturen wurden hier für die Verbesserung des Models miteinbezogen. Die schrittweise Berücksichtigung von Pons und Medulla oblongata erklärte bei SCA3 53\% der Varianz von SARA $[\mathrm{F}(2 ; 21)=11,7 ; \mathrm{p}<0,001]$. Die Varianz der klinischen Symptomatik bei SCA6 wurde zu 82\% durch das Zerebellum und den Pons erklärt $[\mathrm{F}(2 ; 7)=15,9 ; \mathrm{p}=0,003]$. Das statistische Model berücksichtigte für SCA1, SCA3 und SCA6 auch bei veränderten Ein- und Ausschlussbedingungen $\left(\mathrm{p}_{\mathrm{in}}=0,25\right.$ und $\left.\mathrm{p}_{\text {out }}=0,30\right)$ keine weiteren Strukturen.

\subsection{Klassifizierung der Gruppen mit Hilfe der Diskriminanzanalyse}

Die Gruppenunterschiede konnten mit Hilfe der Varianzanalyse quantifiziert werden (Tabelle 4). Aufgrund der breiten Streuung der individuellen Daten war es allerdings nicht möglich, die Gruppenzugehörigkeit unter Zuhilfenahme der Volumetrie einer einzigen anatomischen Struktur festzustellen.

Für die Prognose der Gruppenzugehörigkeit der einzelnen Elemente wurde daher eine lineare schrittweise Diskriminanzanalyse verwendet. Diese Analyse berücksichtigt die einzelnen Merkmalsvariablen nacheinander. Es wurde jeweils diejenige Variable ausgewählt und in die Diskriminanzfunktion mit einbezogen, die für ein bestimmtes Gütema $\beta\left(p_{\text {in }}=0,10 ; p_{\text {out }}=0,25\right)$ maximale Werte zeigte. Durch die Extraktion von sechs Variablen konnten drei kanonische Diskriminanzfunktionen erstellt werden. Die erste kanonische Funktion erklärte $80 \%$, die zweite 14\% und die dritte 6\% der Varianz. Durch die Reihenfolge, mit der die Variablen in die Diskriminanzfunktion aufgenommen wurden, konnte die relative Wichtigkeit der TICV-normalisierten Volumina bestimmt werden: Pons (31\%), Medulla oblongata $(20 \%)$, Vermis (18\%), zerebelläre Hemisphären (15\%), Putamen (12\%) und Mesenzephalon (3\%). 
Tabelle 8 Diagnostische Klassifikationsmatrix basierend auf der MRT-Volumetrie; diese Matrix gibt die Prozentzahlen (absolute Anzahl) der richtigen Gruppenzuordnungen gemäß der genetischen Diagnose durch die Diskriminanzanalyse wieder.

\begin{tabular}{|c|c|c|c|c|c|}
\hline & \multicolumn{5}{|c|}{ Vorhersagewahrscheinlichkeit durch MRT-Volumetrie (n) } \\
\hline \multirow{5}{*}{ 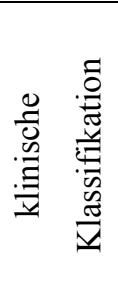 } & & Kontrollpersonen & SCA1 & SCA3 & SCA6 \\
\hline & Kontrollpersonen (31) & $83,9 \%(26)$ & 0 & 0 & $16,1 \%(5)$ \\
\hline & SCA1 (48) & 0 & $77,1 \%(37)$ & $22,9 \%(11)$ & 0 \\
\hline & SCA3 (24) & 0 & $16,7 \%(4)$ & $83,3 \%(20)$ & 0 \\
\hline & SCA6 (10) & 0 & 0 & 0 & $100 \%(10)$ \\
\hline
\end{tabular}

Mit Hilfe der drei Funktionen war eine Klassifizierung der drei Krankheitsgruppen (SCA1, SCA3 und SCA6) mit einer Wahrscheinlichkeit von 82,3\% möglich. Alle zehn SCA6-Patienten konnten richtig klassifiziert werden (Tabelle 8). Lediglich fünf Kontrollpersonen wurden fälschlicherweise der SCA6-Gruppe zugeordnet. Die größte Anzahl an falschen Klassifizierungen trat zwischen SCA1- und SCA3-Patienten auf. Für elf der 48 SCA1-Patienten (22,9\%) wurde eine SCA3-Mutation vorhergesagt. Vier der 24 SCA3-Patienten (16,7\%) wurden als SCA1-Mutation eingeordnet.

Hätte das Untersuchungskollektiv nur aus SCA1- und SCA3-Patienten bestanden, wäre ein wesentlich einfacheres statistisches Model genauso informativ gewesen. Unter Berücksichtigung eines Gütemaßes $\left(\mathrm{p}_{\mathrm{in}}=0,10 ; \mathrm{p}_{\text {out }}=0,25\right)$ ergab die Analyse für die relative Wichtigkeit der TICV-normalisierten Volumina für die Diskriminanzfunktion folgende Werte: zerebelläre Hemisphären (42\%), Mesenzephalon (39\%) und Putamen (19\%). Die resultierende kanonische Trennfunktion lautete:

$$
f_{(1)}=-0,785 \times \text { Mesenzephalon }+0,839 \text { x zerebelläre Hemisphären }+0,383 \times \text { Putamen }
$$

Unter Berücksichtigung dieser Funktion konnten 80,6\% der SCA1- und SCA3-Patienten richtig zugeordnet werden. Von den 48 SCA1-Patienten wurden $37(77,1 \%)$ und von den 24 SCA3-Patienten $21(87,5 \%)$ richtig klassifiziert. Elf der 48 SCA1-Patienten $(22,9 \%)$ wurden fälschlicherweise als SCA3 und drei der 24 SCA3-Patienten (12,5\%) versehentlich als SCA1 diagnostiziert. 


\section{$4 \quad$ Diskussion}

Im Rahmen einer Multizenterstudie konnten wir zeigen, dass bei Patienten mit SCA die MRT mit anschließender Volumetrie eine geeignete Methode ist, um die klinische Dysfunktion und den Krankheitsfortschritt zu dokumentieren. Mit Hilfe der volumetrischen Auswertung von MRT-Datensätzen konnte das Ausmaß der Atrophie der neuroanatomischen Strukturen individuell bestimmt und anschließend mit dem Schweregrad der Ataxie (SARA) verglichen werden.

\subsection{MR-Bildgebung mit anschließender 3D-Volumetrie}

Aufgrund des hohen Auflösungsvermögens ist die MRT für die Beurteilung des Zerebellums, Hirnstamms und Rückenmarks von besonderer Wichtigkeit (Courchesne et al. 1989; Press et al. 1989). Für die dreidimensionale Darstellung neuroanatomischer Strukturen wurde im Rahmen dieser Multizenterstudie für jeden 1,5-TeslaKernspintomographen ein separates MRT-Protokoll (vgl. Abschnitt 2.3.1) erstellt. Dadurch wurde eine besonders hohe Trennschärfe zwischen den einzelnen Hirnregionen gewährleistet. Diese Protokolle basieren auf den Erfahrungen von Luft und seinen Kollegen, die im Rahmen einer Studie für die Optimierung und Validierung der MRTProtokolle Phantomobjekte mit bekanntem Volumen und typischem Gewebekontrast benutzten (Luft et al. 1996).

Das anschließend in dieser Arbeit angewendete Verfahren zur dreidimensionalen Hirnvolumetrie verbindet eine geometrisch standardisierte, interaktive Vorsegmentierung mit einem automatischen Segmentierungsverfahren (Region-growing). Durch die Minimierung des Partialvolumeneffektes besitzt die 3D-Volumetrie für die Quantifizierung der Hirnatrophie im Vergleich zu zweidimensionalen Verfahren ein höheres Maß an Genauigkeit. Dies wird durch folgende Punkte erreicht: (1) Verwendung einer Kopfspule gewährleistet eine standardisierte Position des Probanden, (2) nutzerabhängige Auswahl einer geeigneten MRT-Schicht entfällt, da relevante Strukturen auf allen Schnitten, auf denen sie abgebildet sind, vermessen werden, (3) semiautomatische Segmentierung der relevanten Strukturen ermöglicht ein hohes Maß an Reproduzierbarkeit. 
Die Methode der 3D-Volumetrie wurde bereits erfolgreich für die Gegenüberstellung von Patienten mit SCA1, SCA2 und SCA3 (Klockgether et al. 1998b) sowie bei Patienten mit akinestisch rigiden Bewegungsstörungen (Schulz et al. 1999) eingesetzt. Im Rahmen einer Longitudinalstudie für Patienten mit MSA-C konnte unter Zuhilfenahme der 3D-Volumetrie der Fortschritt der Erkrankung über einen Zeitraum von zwei Jahren dokumentiert werden (Hauser et al. 2006).

Das Atrophiemuster bei Patienten mit SCA ist indes nicht spezifisch für einen einzelnen Genotyp (Döhlinger et al. 2008). Bei unbekanntem Genotyp kann die MRT daher nur eingesetzt werden, um zunächst zwischen OPCA und CCA unterscheiden zu können. Allerdings kann es auch vorkommen, dass Patienten beim erstmaligen Auftreten von Symptomen zunächst nur eine einfach zerebelläre Atrophie und erst im späteren Krankheitsverlauf ein olivo-pontino-zerebelläres Atrophiemuster zeigen. Eine Korrelation zwischen dem Atrophiemuster und der zugrunde liegenden Pathologie kann daher erst für fortgeschrittene Krankheitsstadien angenommen werden.

Der Zeitaufwand für die volumetrische Auswertung eines Datensatzes war mit insgesamt drei Stunden relativ hoch. Aufgrund der fehlenden vollständigen Automatisierung dürfte die 3D-Volumetrie für die Diagnosestellung im klinischen Alltag daher kaum relevant sein. Auch in naher Zukunft kann nicht damit gerechnet werden, dass die Segmentierung aufgrund der individuellen Verteilung anatomischer Landmarken vollständig automatisiert wird (Shin et al. 2000). Darüber hinaus muss man sich der Tatsache bewusst sein, dass durch die ROI-basierte Volumetrie manuell editierte Daten entstehen, für die unter Umständen die Reproduzierbarkeit und die Objektivität nicht gewährleistet werden kann. Daher können alle segmentierten Daten als potentiell fehlerbehaftet betrachtet werden.

Durch die ständige Weiterentwicklung der MRT-Technologie kann für die Zukunft eine größere Verbreitung von 3,0-Tesla-Kernspintomographen angenommen werden. Dies hätte eine höhere Auflösung der MRT-Datensätze zur Folge und würde im Vergleich zu den derzeitigen 1,5-Tesla-Apparaten eine noch bessere Differenzierung der Gewebekontraste ermöglichen. 


\subsection{Demographische und klinische Charakteristika}

Der Krankheitsbeginn in der SCA1-, SCA3- und SCA6-Gruppe der Multizenterstudie stimmt mit den Angaben in der Literatur überein. Während bei SCA1 und SCA3 die ersten Symptome im Mittel zwischen dem 35. und 40. Lebensjahr beobachtet werden, ist der Krankheitsbeginn bei SCA6 fast 20 Jahre später (Klockgether et al. 1998a; Stevanin et al. 1997; Schöls et al. 1997; van de Warrenburg et al. 2005). Im Vergleich zu SCA1- und SCA3- wiesen die SCA6-Patienten dieser Multizenterstudie ein wesentlich höheres Alter bei Auftreten der ersten Symptome auf(Tabelle 3).

Bürk und ihre Kollegen berichten über die physiologischen und die expandierten Repeatzahlen bei SCA1, SCA3 und SCA6 (Bürk et al. 1999). Alle im Rahmen dieser Arbeit bestimmten CAG-Repeatlängen der Patienten mit SCA1, SCA3 oder SCA6 entsprechen diesen Erkenntnissen (Vergleich von Tabelle 1 mit Tabelle 3). In Hinblick auf die klinische Symptomatik, die mit Hilfe von SARA gemessen wurde, waren die drei SCA-Gruppen nahezu ausgeglichen (Tabelle 3).

\subsection{Neuroanatomische Veränderungen bei SCA1, SCA3 und SCA6}

Um das Ausmaß der Atrophie zu quantifizieren, wurde in dieser Multizenterstudie bei SCA6-Patienten das erste Mal die MRT mit anschließender 3D-Volumetrie eingesetzt. Bei SCA1- und SCA3-Patienten hingegen wurde die 3D-Volumetrie bereits erstmalig durch Klockgether und seine Kollegen angewendet (Klockgether et al. 1998b).

Bei allen drei Genotypen konnte mit Hilfe der 3D-Volumetrie ein bestimmtes Atrophiemuster mit spezifischen Charakteristika beobachtet werden. Das Muster der neurodegenerativen Veränderungen bei SCA6, das fast ausschließlich auf den zerebellären Kortex beschränkt war, unterschied sich grundlegend von dem bei SCA1 und SCA3. Auch wenn nicht so stark betont wie bei SCA1 und SCA3, so wurden auch für SCA6 atrophische Veränderungen des Pons und des gesamten Hirnstamms gefunden. Wie bereits in einer früheren Monozenterstudie (Klockgether et al. 1998b) beobachtet werden konnte, zeigte sich auch in der vorliegenden Multizenterstudie bei SCA1 und 
SCA3 eine substanzielle Atrophie des Nucleus caudatus und des Putamens. Bei SCA6 konnten hingegen konnten keine neurodegenerativen Veränderungen der Basalganglien nachgewiesen werden.

\subsection{Klinisch-neuroanatomische Korrelationen und Krankheitsverlauf}

Es wäre wünschenswert, wenn die MRT mit anschließender 3D-Volumetrie als aussagekräftiger Biomarker für den Krankheitsverlauf und den klinischen Schweregrad von SCA angesehen werden könnte. Um dies zu gewährleisten, sollte das Ausmaß der neurodegenerativen Veränderungen mit dem Schweregrad der Ataxie, den molekularen Biomarkern, wie bspw. der CAG-Repeatlänge, und der Erkrankungsdauer korrelieren.

Durch den Einsatz der neu entwickelten SARA konnte im Rahmen dieser Multizenterstudie erstmalig für SCA1, SCA3 und SCA6 gezeigt werden, dass der Schweregrad der Ataxie mit der Atrophie einzelner neuroanatomischer Strukturen korreliert. Für SCA1 bestand die größte Korrelation mit dem Volumen der Pons, für SCA3 mit dem Volumen des Hirnstamms und für SCA6 mit dem Volumen des Zerebellums. Der relativ hohe Wert für die Korrelation zwischen der Atrophie der Medulla oblongata und SARA bei SCA6 (vgl. Tabelle 5: -0,625) darf nicht überinterpretiert werden. Er ist in erster Linie durch die Messmethode, die mittleren Kleinhirnstiele wurden bei der Berechnung des Hirnstammvolumens mitberücksichtigt, bedingt.

Eine frühere Studie berichtet von einem Zusammenhang der CAG-Repeatlänge mit den 2D-Abmessungen des Pons und des Vermis bei SCA3-Patienten (Onodera et al. 1998). Mit Hilfe der 3D-Volumetrie konnte für eine Kohorte von elf SCA3-Patienten ein solcher Zusammenhang nicht bestätigt werden (Klockgether et al. 1998b). Im Gegensatz zu diesen Erkenntnissen von Klockgether und seinen Kollegen und wahrscheinlich begünstigt durch eine mehr als doppelt so große Kohorte konnte in der vorliegenden Multizenterstudie für SCA3 eine Korrelation zwischen der Atrophie des Hirnstamms und Nucleus caudatus mit der CAG-Repeatlänge nachgewiesen werden. Für SCA6 war dies, bedingt durch die geringe Spannweite der CAG-Repeatlängen, allerdings nicht möglich. Der fehlende Zusammenhang für SCA1 hingegen bleibt unerklärt. 
Die Korrelation von SARA mit der CAG-Repeatlänge war bei SCA1 sowohl im Untersuchungskollektiv dieser Multizenterstudie als auch in der größeren EuroSCA Kohorte (Schmitz-Hübsch et al. 2008) im Vergleich zu SCA3 schwächer. Dieser Zusammenhang erklärt für SCA1 - verglichen mit SCA3 - den scheinbar geringeren Einfluss der CAG-Repeatlänge auf den Schweregrad der Ataxie und das Ausmaß der Atrophie.

Der Einfluss der Erkrankungsdauer auf das Ausmaß der Atrophie war insgesamt gering. Für SCA1 korrelierte das Volumen des Pons und für SCA6 das Volumen des Zerebellums mit der Dauer der Erkrankung. Für SCA3 hingegen konnte kein signifikanter Zusammenhang beobachtet werden.

Durch die schrittweise lineare Regressionsanalyse konnte gezeigt werden, dass die Atrophie der einzelnen neuroanatomischen Strukturen jeweils einen Beitrag für den Schweregrad der Ataxie, quantifiziert durch SARA, leistete. Obwohl das Hauptcharakteristikum der drei SCA-Genotypen die Ataxie ist und SARA geeignet ist, den Schweregrad von Ataxie zu quantifizieren, war dieser Anteil jeweils sehr unterschiedlich. Er variierte von $82 \%$ bei SCA6, über $52 \%$ bei SCA3 bis zu nur noch $28 \%$ bei SCA1. Hierfür gibt es mehrere mögliche Gründe: (1) Ataxie ist die Folge von Neurodegeneration und der anschließenden Atrophie der Systeme, die mit dem Zerebellum in Verbindung stehen, einschließlich der zerebellären Efferenzen und Afferenzen. Es wäre denkbar, dass Ataxie durch die Dysfunktion dieser extrazerebellären Systeme bedingt ist, ohne dass dort atrophische Veränderungen beobachtet werden können bzw. diese erst mit einer Verzögerung auftreten. (2) Andererseits wäre es aber auch möglich, dass die Atrophie initial durch synaptische Anpassungsreaktionen, die durch die MRT der einzelnen neuroanatomischen Strukturen nicht abgebildet werden kann, kompensiert wird. (3) SARA misst den Schweregrad von Ataxie. Bei SCA6 wird SARA daher gut durch die Neurodegeneration des Zerebellums erklärt. Andererseits leisten bei SCA3 und insbesondere bei SCA1 die extrazerebellären Manifestationen, z.B. die periphere Neuropathie oder die Degeneration der Hinterstrangbahnen, ebenfalls einen Beitrag zur, durch SARA quantifiziert, klinischen Symptomatik. Die anatomischen Substrate dieser extrazerebellären Symptome werden aber durch die MRT gar nicht berücksichtigt. 


\subsection{Prädiktive Statistik}

Im Gegensatz zur eindeutigen Prognose der Gruppenzugehörigkeit bei SCA6, war die Klassifikation bei SCA1 und SCA3 unter Zuhilfenahme der Volumetrie einer einzigen anatomischen Struktur nicht möglich. Eine lineare schrittweise Diskriminanzanalyse erreichte unter Berücksichtigung der volumetrischen Daten von Pons, Medulla oblongata, Vermis, zerebellären Hemisphären, Putamen und Mesenzephalon eine Klassifizierung der drei Krankheitsgruppen mit einer Wahrscheinlichkeit von 82,3\%.

Das der Klassifizierung zugrunde liegende statistische Rechenmodell unterlag allerdings einer Einschränkung. Normalerweise wird für die Erstellung der Diskriminanzfunktion die untersuchte Gruppe in zwei Stichproben unterteilt. Zunächst wird mit Hilfe der ersten Stichprobe das Rechenmodell erstellt und anschließend wird es durch die zweite Stichprobe validiert. Da es sich bei der SCA um eine seltene Erkrankung handelt und infolgedessen die untersuchte Gruppe von vorneherein sehr klein war, wären bei einer Zweiteilung die Stichproben zu klein geworden. Daher wurden für die Erstellung und Validierung des Rechenmodells identische Stichproben verwendet.

Anhand des individuellen neuropathologischen Atrophiemuster gelang mit Hilfe der Diskriminanzanalyse die Klassifizierung der SCA1-, SCA3- und SCA6-Patienten mit einer relativ hohen Wahrscheinlichkeit (82,3\%). Auch wenn dieses Verfahren keine zu $100 \%$ richtige Prognose der Gruppenzugehörigkeit ermöglichte, so war es immer noch wesentlich effizienter als die alleinige volumetrische Auswertung einzelner neuroanatomischer Strukturen. 


\subsection{Schlussfolgerung und Ausblick}

Die MRT mit anschließender 3D-Volumetrie ermöglichte im Rahmen einer Multizenterstudie für Patienten mit SCA1, SCA3 oder SCA6 die Identifizierung spezifischer neuroanatomischer Regionen, die als geeignete Biomarker für die Charakterisierung der neuropathologischen Veränderungen angesehen werden können.

Für SCA6 sind das Zerebellum und die zerebellären Hemisphären von besonderer Relevanz. Die Volumina dieser zwei Strukturen zeigten eine hohe Korrelation mit dem klinischen Schweregrad der Erkrankung und einen schwachen Zusammenhang mit der Erkrankungsdauer. Für SCA3 wurden der Hirnstamm bzw. die Volumina des Pons und des Mesenzephalons als besonders wichtige Strukturen identifiziert. Hier bestand sowohl eine Korrelation mit dem Schweregrad der Ataxie als auch mit der CAG-Repeatlänge des expandierten Allels. Für SCA1 ist das Volumen der Pons von großer Wichtigkeit. Dieses Volumen zeigte eine gute Korrelation mit den klinischen Symptomen und einen schwachen Zusammenhang mit der Erkrankungsdauer und der CAG-Repeatlänge.

Die Volumina dieser neuroanatomischen Strukturen zeigten im Rahmen dieser Querschnittsstudie gute Korrelationen mit dem klinischen Schweregrad der Erkrankung, der CAG-Repeatlänge und / oder der Erkrankungsdauer. Ob die genannten Strukturen tatsächlich potente Biomarker für das Monitoring des Krankheitsverlaufs und die Wirksamkeit interventioneller Therapie sind, sollte im Rahmen einer Longitudinalstudie weiter untersucht werden. Ferner könnten aus einer solchen Studie auch Informationen darüber gewonnen werden, ob das Fortschreiten der neurodegenerativen Veränderungen bei SCA im Verlauf konstant ist oder in Abhängigkeit vom Krankheitsstadium variiert. 


\section{$5 \quad$ Zusammenfassung}

Die autosomal-dominant vererbten spinozerebellären Ataxien (SCA) bilden eine heterogene Gruppe, die klinisch charakterisiert ist durch eine langsam progressive Ataxie und - in Abhängigkeit vom Genotyp - zusätzliche extrazerebelläre Symptome. Biomarker für das Monitoring der neurologischen Dysfunktion existieren derzeit noch nicht. Beurteilungsskalen für die Quantifizierung der klinischen Symptome konnten erst kürzlich validiert werden. Um die Atrophie definierter neuroanatomischer Regionen zu quantifizieren, wurde bei 82 Patienten mit SCA1, SCA3 oder SCA6 sowie bei 31 Kontrollpersonen nach vorangehender Bildgebung mittels Magnetresonanztomographie (MRT) eine anschließende quantitative 3D-Volumetrie durchgeführt.

Diese Untersuchung ergab für SCA1 und SCA3 schwere Atrophien des Pons, der Medulla oblongata, der zerebellären Hemisphären, des Vermis, des Putamens und des Nucleus caudatus. Bei SCA6 hingegen konnten atrophische Veränderungen nur für das Zerebellum und den Pons nachgewiesen werden. Für den zerebralen Kortex konnte keine wesentliche Atrophie beobachtet werden. Mit Hilfe der volumetrischen Auswertung einzelner anatomischer Regionen war es nicht möglich, SCA1, SCA3, SCA6 und die Kontrollpersonen untereinander $\mathrm{zu}$ differenzieren. Dagegen erreichte eine Diskriminanzanalyse unter Berücksichtigung der zuvor gewonnenen Daten über Pons, Vermis, zerebelläre Hemisphären, Medulla oblongata, Mesenzephalon und Putamen eine Reklassifizierungswahrscheinlichkeit von 82,3\%. Die Repeatlängen des expandierten Allels zeigten eine negative Korrelation mit den Volumina von Mesenzephalon, Pons, Nucleus caudatus und Putamen bei SCA3, eine nur schwache Korrelation mit dem Pons bei SCA1 und indessen keine Korrelation bei SCA6. Der Schweregrad der Ataxie, quantifiziert durch SARA (Scale for the assessment and rating of ataxia), korrelierte bei SCA1 und SCA3 mit der Atrophie der Hirnstammstrukturen, insbesondere mit dem Pons, und zeigte keine Zusammenhänge mit den atrophischen Veränderungen der zerebellären Hemisphären und des Vermis. Für SCA6 wurde lediglich eine Korrelation mit der Atrophie des Zerebellums beobachtet.

Die Erkenntnisse dieser Arbeit unterstreichen den Stellenwert der MRT für die Beurteilung des klinischen Verlaufs von SCA. Ferner scheint das klinische Erscheinungsbild der Genotypen durch unterschiedliche pathoanatomische Ursachen bedingt zu sein. 


\section{$6 \quad$ Literaturverzeichnis}

Abele M, Bürk K, Laccone F, Dichgans J, Klockgether T (2001): Restless legs syndrome in spinocerebellar ataxia types 1, 2, and 3. J Neurol 248, 311-314

Bamford KA, Caine ED, Kido DK, Cox C, Shoulson I (1995): A prospective evaluation of cognitive decline in early Huntington's disease: functional and radiographic correlates. Neurology $\underline{45}, 1867-1873$

Bradley WG, Waluch V, Yadley RA, Wycoff RR (1984): Comparison of CT and MR in 400 patients with suspected disease of the brain and cervical spinal cord. Radiology $\underline{152}, 695-702$

Brant-Zawadzki M, Davis PL, Crooks LE, Mills CM, Norman D, Newton TH, Sheldon P, Kaufman L (1983): NMR demonstration of cerebral abnormalities: comparison with CT. AJR Am J Roentgenol 140, 847-854

Bürk K, Abele M, Fetter M, Dichgans J, Skalej M, Laccone F, Didierjean O, Brice A, Klockgether T (1996): Autosomal dominant cerebellar ataxia type I clinical features and MRI in families with SCA1, SCA2 and SCA3. Brain $\underline{119}$, 1497-1505

Bürk K, Klockgether T, Dichgans J (1999): New insights in the molecular genetics and pathophysiology of hereditary ataxias. Nervenarzt $\underline{70}, 491-495$

Butteriss D, Chinnery P, Birchall D (2005): Radiological characterization of spinocerebellar ataxia type 6 . Br J Radiol 78, 694-696

Bydder GM, Steiner RE, Young IR, Hall AS, Thomas DJ, Marshall J, Pallis CA, Legg NJ (1982): Clinical NMR imaging of the brain: 140 cases. AJR Am J Roentgenol 139, 215-236

Cohen J: Statistical power analysis for the behavioral sciences, 2. Auflage. Hillsdale: Lawrence Erlbaum Associates, New York 1988

Courchesne E, Press GA, Murakami J, Berthoty D, Grafe M, Wiley CA, Hesselink JR (1989): The cerebellum in sagittal plane--anatomic-MR correlation: 1 . The vermis. AJR Am J Roentgenol 153, 829-835

Döhlinger S, Hauser TK, Borkert J, Luft AR, Schulz JB (2008): Magnetic resonance imaging in spinocerebellar ataxias. Cerebellum $\underline{7}, 204-214$

Eritaia J, Wood SJ, Stuart GW, Bridle N, Dudgeon P, Maruff P, Velakoulis D, Pantelis C (2000): An optimized method for estimating intracranial volume from magnetic resonance images. Magn Reson Med 44, 973-977

Fox NC, Schott JM (2004): Imaging cerebral atrophy: normal ageing to Alzheimer's disease. Lancet $\underline{363}, 392-394$ 
Fox NC, Crum WR, Scahill RI, Stevens JM, Janssen JC, Rossor MN (2001): Imaging of onset and progression of Alzheimer's disease with voxel-compression mapping of serial magnetic resonance images. Lancet $\underline{358}, 201-205$

Greenfield JG: The spinocerebellar degenerations. Charles C. Thomas, Springfield (Illinois) 1954

Gröschel K, Hauser TK, Luft A, Patronas N, Dichgans J, Litvan I, Schulz JB (2004): Magnetic resonance imaging-based volumetry differentiates progressive supranuclear palsy from corticobasal degeneration. Neuroimage 21, 714-724

Guerrini L, Lolli F, Ginestroni A, Belli G, Della Nave R, Tessa C, Foresti S, Cosottini M, Piacentini S, Salvi F et al. (2004): Brainstem neurodegeneration correlates with clinical dysfunction in SCA1 but not in SCA2. A quantitative volumetric, diffusion and proton spectroscopy MR study. Brain $\underline{127}, 1785-1795$

Harding AE (1982): The clinical features and classification of the late onset autosomal dominant cerebellar ataxias. A study of 11 families, including descendants of the 'the Drew family of Walworth'. Brain 105, 1-28

Hauser TK, Luft A, Skalej M, Nägele T, Kircher TT, Leube DT, Schulz JB (2006): Visualization and quantification of disease progression in multiple system atrophy. Mov Disord 21, 1674-1681

Holmes G (1908): An attempt to classify cerebellar disease, with a note on Marie's hereditary cerebellar ataxia. Brain $\underline{30}, 545-567$

Kassubek J, Juengling FD, Kioschies T, Henkel K, Karitzky J, Kramer B, Ecker D, Andrich J, Saft C, Kraus P et al. (2004): Topography of cerebral atrophy in early Huntington's disease: a voxel based morphometric MRI study. J Neurol Neurosurg Psychiatry $\underline{75}, 213-220$

Klockgether T: Spinocerebellar Ataxia 1. In: Neurological Disease and Therapy. Dekker M., Inc., New York 1999, 343-361

Klockgether T (2005): Ataxias. Diagnostic procedure and treatment. Nervenarzt $\underline{76}$, $1275-1283$

Klockgether T (2008): The clinical diagnosis of autosomal dominant spinocerebellar ataxias. Cerebellum ㄱ, 101-105

Klockgether T, Ludtke R, Kramer B, Abele M, Bürk K, Schöls L, Riess O, Laccone F, Boesch S, Lopes-Cendes I et al. (1998a): The natural history of degenerative ataxia: a retrospective study in 466 patients. Brain $\underline{121}, 589-600$

Klockgether T, Skalej M, Wedekind D, Luft AR, Welte D, Schulz JB, Abele M, Bürk K, Laccone F, Brice A et al. (1998b): Autosomal dominant cerebellar ataxia type I. MRI-based volumetry of posterior fossa structures and basal ganglia in spinocerebellar ataxia types 1, 2 and 3. Brain $\underline{121}, 1687-1693$ 
Lauterbur PC (1973): Image formation by induced local interactions. Examples employing NMR. Nature 242, 190

Lima L, Coutinho P (1980): Clinical criteria for diagnosis of Machado-Joseph disease: report of a non-Azorena Portuguese family. Neurology 30, 319-322

Luft AR, Skalej M, Welte D, Kolb R, Klose U (1996): Reliability and exactness of MRI-based volumetry: a phantom study. J Magn Reson Imaging $\underline{6}$, 700-704

Luft AR, Skalej M, Welte D, Kolb R, Bürk K, Schulz JB, Klockgether T, Voigt K (1998): A new semiautomated, three-dimensional technique allowing precise quantification of total and regional cerebellar volume using MRI. Magn Reson Med 40, 143-151

Murata Y, Yamaguchi S, Kawakami H, Imon Y, Maruyama H, Sakai T, Kazuta T, Ohtake T, Nishimura M, Saida T et al. (1998a): Characteristic magnetic resonance imaging findings in Machado-Joseph disease. Arch Neurol 55, 33-37

Murata Y, Kawakami H, Yamaguchi S, Nishimura M, Kohriyama T, Ishizaki F, Matsuyama Z, Mimori Y, Nakamura S (1998b): Characteristic magnetic resonance imaging findings in spinocerebellar ataxia 6. Arch Neurol 55, 1348-1352

Nabatame H, Fukuyama H, Akiguchi I, Kameyama M, Nishimura K, Nakano Y (1988): Spinocerebellar degeneration: qualitative and quantitative MR analysis of atrophy. $\mathrm{J}$ Comput Assist Tomogr 12, 298-303

Nicoletti G, Lodi R, Condino F, Tonon C, Fera F, Malucelli E, Manners D, Zappia M, Morgante L, Barone P et al. (2006): Apparent diffusion coefficient measurements of the middle cerebellar peduncle differentiate the Parkinson variant of MSA from Parkinson's disease and progressive supranuclear palsy. Brain $\underline{129}$, 2679-2687

Onodera O, Idezuka J, Igarashi S, Takiyama Y, Endo K, Takano H, Oyake M, Tanaka H, Inuzuka T, Hayashi T et al. (1998): Progressive atrophy of cerebellum and brainstem as a function of age and the size of the expanded CAG repeats in the MJD1 gene in Machado-Joseph disease. Ann Neurol 43, 288-296

Orr HT, Chung MY, Banfi S, Kwiatkowski TJ, Jr., Servadio A, Beaudet AL, McCall AE, Duvick LA, Ranum LP, Zoghbi HY (1993): Expansion of an unstable trinucleotide $\mathrm{CAG}$ repeat in spinocerebellar ataxia type 1 . Nat Genet $\underline{4}, 221-226$

Press GA, Murakami J, Courchesne E, Berthoty DP, Grafe M, Wiley CA, Hesselink JR (1989): The cerebellum in sagittal plane--anatomic-MR correlation: 2 . The cerebellar hemispheres. AJR Am J Roentgenol 153, 837-846

Purcell EM, Torry MC, Pound RV (1946): Resonance absorption by nuclear magnetic moments in a solid. Phys Rev $\underline{69}, 37-38$

Rosas HD, Goodman J, Chen YI, Jenkins BG, Kennedy DN, Makris N, Patti M, Seidman LJ, Beal MF, Koroshetz WJ (2001): Striatal volume loss in HD as measured by MRI and the influence of CAG repeat. Neurology 57, 1025-1028 
Sasaki H, Fukazawa T, Yanagihara T, Hamada T, Shima K, Matsumoto A, Hashimoto K, Ito N, Wakisaka A, Tashiro K (1996): Clinical features and natural history of spinocerebellar ataxia type 1. Acta Neurol Scand $\underline{93}, 64-71$

Satoh JI, Tokumoto H, Yukitake M, Matsui M, Matsuyama Z, Kawakami H, Nakamura S, Kuroda Y (1998): Spinocerebellar ataxia type 6: MRI of three Japanese patients. Neuroradiology $\underline{40}, 222-227$

Schmitz-Hübsch T, Tezenas du Montcel S, Baliko L, Boesch S, Bonato S, Fancellu R, Giunti P, Globas C, Kang JS, Kremer B et al. (2006a): Reliability and validity of the International Cooperative Ataxia Rating Scale: a study in 156 spinocerebellar ataxia patients. Mov Disord 21, 699-704

Schmitz-Hübsch T, du Montcel ST, Baliko L, Berciano J, Boesch S, Depondt C, Giunti P, Globas C, Infante J, Kang JS et al. (2006b): Scale for the assessment and rating of ataxia: development of a new clinical scale. Neurology $\underline{66}, 1717-1720$

Schmitz-Hübsch T, Coudert M, Bauer P, Giunti P, Globas C, Baliko L, Filla A, Mariotti C, Rakowicz M, Charles P et al. (2008): Spinocerebellar ataxia types 1, 2, 3, and 6: disease severity and nonataxia symptoms. Neurology $\underline{71}, 982-989$

Schocke MF, Seppi K, Esterhammer R, Kremser C, Mair KJ, Czermak BV, Jaschke W, Poewe W, Wenning GK (2004): Trace of diffusion tensor differentiates the Parkinson variant of multiple system atrophy and Parkinson's disease. Neuroimage 21, 1443-1451

Schoenberg BS (1978): Epidemiology of the inherited ataxias. Adv Neurol 21, 15-32

Schöls L, Amoiridis G, Buttner T, Przuntek H, Epplen JT, Riess O (1997): Autosomal dominant cerebellar ataxia: phenotypic differences in genetically defined subtypes? Ann Neurol $\underline{42}, 924-932$

Schöls L, Kruger R, Amoiridis G, Przuntek H, Epplen JT, Riess O (1998a): Spinocerebellar ataxia type 6: genotype and phenotype in German kindreds. J Neurol Neurosurg Psychiatry $\underline{64}, 67-73$

Schöls L, Haan J, Riess O, Amoiridis G, Przuntek H (1998b): Sleep disturbance in spinocerebellar ataxias: is the SCA3 mutation a cause of restless legs syndrome? Neurology $\underline{51}, 1603-1607$

Schöls L, Bauer P, Schmidt T, Schulte T, Riess O (2004): Autosomal dominant cerebellar ataxias: clinical features, genetics, and pathogenesis. Lancet Neurol $\underline{3}$, 291-304

Schulz JB, Skalej M, Wedekind D, Luft AR, Abele M, Voigt K, Dichgans J, Klockgether T (1999): Magnetic resonance imaging-based volumetry differentiates idiopathic Parkinson's syndrome from multiple system atrophy and progressive supranuclear palsy. Ann Neurol 45, 65-74

Shin H, Stamm G, Hogemann D, Galanski M (2000): Basic principles of data acquisition and data processing for construction of high quality virtual models. Radiologe $\underline{40}$, 304-312 
Specht K, Minnerop M, Muller-Hubenthal J, Klockgether T (2005): Voxel-based analysis of multiple-system atrophy of cerebellar type: complementary results by combining voxel-based morphometry and voxel-based relaxometry. Neuroimage $\underline{25}, 287-293$

Stevanin G, Durr A, David G, Didierjean O, Cancel G, Rivaud S, Tourbah A, Warter JM, Agid Y, Brice A (1997): Clinical and molecular features of spinocerebellar ataxia type 6. Neurology $\underline{49}, 1243-1246$

Stevanin G, Durr A, Brice A (2000): Clinical and molecular advances in autosomal dominant cerebellar ataxias: from genotype to phenotype and physiopathology. Eur $\mathrm{J}$ Hum Genet $\underline{8}, 4-18$

St George-Hyslop P, Rogaeva E, Huterer J, Tsuda T, Santos J, Haines JL, Schlumpf K, Rogaev EI, Liang Y, McLachlan DR et al. (1994): Machado-Joseph disease in pedigrees of Azorean descent is linked to chromosome 14. Am J Hum Genet 55, 120-125

Subramony SH, May W, Lynch D, Gomez C, Fischbeck K, Hallett M, Taylor P, Wilson R, Ashizawa T (2005): Measuring Friedreich ataxia: Interrater reliability of a neurologic rating scale. Neurology $\underline{64}, 1261-1262$

Takiyama Y, Nishizawa M, Tanaka H, Kawashima S, Sakamoto H, Karube Y, Shimazaki H, Soutome M, Endo K, Ohta S et al. (1993): The gene for Machado-Joseph disease maps to human chromosome 14q. Nat Genet $\underline{4}, 300-304$

Talairach J, Tournoux P: Co-Planar Stereotaxic Atlas of the Human Brain: 3Dimensional Proportional System: An Approach to Cerebral Imaging. Thieme, New York 1988

Vahedi K, Joutel A, Van Bogaert P, Ducros A, Maciazeck J, Bach JF, Bousser MG, Tournier-Lasserve E (1995): A gene for hereditary paroxysmal cerebellar ataxia maps to chromosome 19p. Ann Neurol 37, 289-293

van de Warrenburg BP, Hendriks H, Durr A, van Zuijlen MC, Stevanin G, Camuzat A, Sinke RJ, Brice A, Kremer BP (2005): Age at onset variance analysis in spinocerebellar ataxias: a study in a Dutch-French cohort. Ann Neurol 57, 505-512

Whitwell JL, Crum WR, Watt HC, Fox NC (2001): Normalization of cerebral volumes by use of intracranial volume: implications for longitudinal quantitative MR imaging. Am J Neuroradiol 22, 1483-1489

Yabe I, Sasaki H, Yamashita I, Takei A, Tashiro K (2001): Clinical trial of acetazolamide in SCA6, with assessment using the Ataxia Rating Scale and body stabilometry. Acta Neurol Scand 104, 44-47

Ying SH, Choi SI, Perlman SL, Baloh RW, Zee DS, Toga AW (2006): Pontine and cerebellar atrophy correlate with clinical disability in SCA2. Neurology $\underline{66}, 424-426$

Zhuchenko O, Bailey J, Bonnen P, Ashizawa T, Stockton DW, Amos C, Dobyns WB, Subramony SH, Zoghbi HY, Lee CC (1997): Autosomal dominant cerebellar ataxia (SCA6) associated with small polyglutamine expansions in the alpha 1A-voltagedependent calcium channel. Nat Genet $\underline{15}, 62-69$ 


\section{Danksagung}

Mein besonderer Dank gilt meinem Doktorvater Herrn Prof. Dr. med. Jörg B. Schulz für die freundliche Vergabe des Themas und die stetige Unterstützung der Arbeit. Seine vielfältigen Anregungen und hilfreichen Kommentare waren für mich sehr wertvoll.

Herrn Dr. med. Till-Karsten Hauser in Tübingen danke ich, dass er mich mit der Auswertung der kernspintomographischen 3D-Datensätze vertraut gemacht hat. Dies war eine wichtige Grundlage für die vorliegende Arbeit.

Außerdem möchte ich mich bei Herrn Dr. med. Klaus Gröschel, der mir in Göttingen für die Beantwortung computertechnischer Fragen zur Verfügung stand, bedanken.

Für die Kommunikation mit den Zentren des europäischen Konsortiums EuroSCA und die Disposition der Datensätze danke ich Frau Cathy Ludwig.

Frau Dipl.-Psychologin Stefanie Wolf danke ich für die tatkräftige Unterstützung bei der statistischen Auswertung der Daten.

Bei meinen Schwestern Franziska Deichstetter und Susanne Borkert möchte ich mich für das gewissenhafte Korrekturlesen dieser Arbeit bedanken. 